\title{
A generalized cognitive decline (GCoDe) partly mediates the effects of neuro-immune and neuro-oxidative toxicity on the symptomatome and lowered quality of life in (deficit) schizophrenia.
}

\author{
Michael Maes, M.D., Ph.D. ${ }^{\text {a b,c }}$, Buranee Kanchanatawan, M.D. ${ }^{a}$ \\ a Department of Psychiatry, Faculty of Medicine, Chulalongkorn University, Bangkok, \\ Thailand \\ e Department of Psychiatry, Medical University of Plovdiv, Plovdiv, Bulgaria

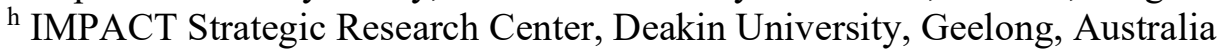

Corresponding author:

Prof. Dr. Michael Maes, M.D., Ph.D.

IMPACT Strategic Research Center

Barwon Health

Deakin University

Geelong, Vic

Australia

dr.michaelmaes@hotmail.com

https://scholar.google.co.th/citations?user=1wzMZ7UAAAAJ\&hl=th\&oi=ao

\begin{abstract}
Recently we showed that schizophrenia and, especially, deficit schizophrenia is accompanied by neurocognitive impairments as measured with different cognitive batteries. The aim of this study was to examine whether a single trait underpins aberrations in 9 key Cambridge Neuropsychological Test Automated Battery (CANTAB) probes, verbal fluency (VFT), world list memory (WLM), true recall, and the Mini Mental State Examination (MMSE). We recruited 80 patients with schizophrenia and 40 healthy controls. All patients were assessed using CANTAB tests, namely paired-association learning (PAL), rapid visual information (RVP), spatial working memory (SWM), one touch stocking (OTS), intra/extradimensional set shifting (IED), and emotional recognition test (ERT). We found that a single latent trait, which is essentially unidimensional, underlies the CANTAB tests, VFT, WLM, True Recall and MMSE. The latent trait shows excellent psychometric properties and fits a reflective model and, therefore, reflects a generalized cognitive decline (GCoDe), which is the cause of aberrations in semantic and episodic memory, recall, executive functions, strategy use, rule acquisition, visual sustained attention, attention set-shifting and emotional recognition. $40.5 \%$ of the variance in the GCode is explained by CCL11, IgA to tryptophan catabolites, and increased oxidative toxicity. GCoDe explains $44.8 \%$ of the variance in a single latent trait extracted from psychosis, hostility, excitation, mannerism, negative symptoms, formal thought disorders, and psychomotor retardation and $40.9 \%$ in quality of life scores. GCoDe is significantly greater in deficit than in nondeficit schizophrenia. In conclusion, a GCoDe mediates the effects of neuro-immune and neuro-oxidative toxicity on the phenome of (deficit) schizophrenia.
\end{abstract}

Key words: deficit schizophrenia, cognition, tryptophan catabolites, neuro-immune, oxidative stress, antioxidants, CCL11 


\section{INTRODUCTION}

Recently, we have shown that the phenome of schizophrenia comprises three major components, namely the a) symptomatome comprising psychosis, hostility, excitation, mannerism, and negative (PHEMN) symptoms, psychomotor retardation (PMR), and formal thought disorders (FTD), b) the cognitome, namely the aggregate of cognitive dysfunctions (Maes et al., 2020d), and c) the phenomenome or the self-experiences of the illness as experienced by the patient (Maes et al., 2020d). Furthermore, we showed that a common latent trait, which is essentially unidimensional, underlies PHEMN, PMR and FTD and, therefore, the latter are reflective manifestations of a single underlying construct, namely overall severity of schizophrenia (OSOS) (Maes et al., 2019b; 2020d; Almulla et al., 2020).

Aberrations in the phenomenome consist of lowered self-rated, health-related quality of life (HR-QoL) with lowered scores on the physical health, psychological, social, and environmental health subdomains (Kanchanatawan et al., 2019; Maes et al., 2020d). Aberrations in the cognitome comprise deficits in semantic and episodic memory, true recall, paired association learning, working memory, executive functions, set shifting, and attention (Kanchanatawan et al., 2018d; Kanchanatawan and Maes, 2018; Maes et al., 2020c; 2020d). Furthermore, these cognitome dysfunctions are strongly related with the symptomatome of schizophrenia and, therefore, may play a role in the onset and maintenance of the symptomatome which together may cause lowered HR-QoL (Maes et al., 2020d).

The aberrations in the cognitome, symptomatome, and phenomenome are more pronounced in deficit schizophrenia than in non-deficit schizophrenia (Kanchanatawan et al., 2018b; Maes et al., 2019b; 2020d). The deficit syndrome or major neurocognitive psychosis is classically conceptualized as a subtype of schizophrenia which is characterized by the presence of negative symptoms with a significant functional impact on HR-QoL (Kanchanatawan et al., 2018c; 2019; Maes et al., 2020c). Nevertheless, we found that deficit schizophrenia is not only characterized by increased severity of negative but also by increased severity of PHEM symptoms, PMR and FTD (Kanchanatawan et al, 2018b; 2018c; Almulla et al., 2020).

The neurocognitive dysfunctions in (deficit) schizophrenia can adequately be assessed using cognitive tests, such as the Consortium to Establish a Registry for Alzheimer's disease (CERAD (CERAD, 1986; Kanchanatawan et al., 2018a), the Brief Assessment of Cognition in Schizophrenia (BACS) (Al-Hakeim et al., 2020), and the computerized Cambridge Neuropsychological Test Automated Battery (CANTAB) (CANTAB, 2018; Kanchanatawan et al., 2018b; 2018d; Kanchanatawan and Maes, 2018). Nevertheless, it remains unknown whether the impairments in attention, semantic and episodic memory, working memory, executive functions, planning and emotional recognition are distinct features of schizophrenia or whether they are intertwined manifestations of a single latent trait reflecting a more "generalized cognitive decline" (GCoDe).

We also reported that a large part of the variance in a latent vector extracted from PHEMN symptoms, PMR, FTD, and CERAD tests of semantic and episodic memory and recall, could mechanistically be explained by the effects of multiple neurotoxic pathways (Maes et al., 2020a; 2020b; 2020c). Thus, the latter authors reported that the cognitome and symptomatome of schizophrenia are strongly associated with a) increased oxidative stress toxicity (OSTOX) as indicated by a composite score comprising lipid hydroperoxides $(\mathrm{LOOH})$, malondialdehyde (MDA), and advanced oxidation protein products (AOPP); b) lowered antioxidant defenses as indicated by a composite score comprising paraoxonase (PON)1 activity, sulfhydryl (-SH) groups, and total radical trapping parameter (TRAP) (Maes et al., 2020c); c) increased levels of the neurotoxic and anti-neurogenesis chemokine (C-C motif) ligand 11 (CCL11) or eotaxin (Sirivichayakul et al., 2019a; 2019b); and d) increased levels of IgA levels directed to neurotoxic tryptophan catabolites (TRYCATS including 
xanthurenic acid, picolinic acid, and 3-OH-kynurenine) (Kanchanatawan et al., 2018a). Activation of the TRYCAT pathway is not only significantly associated with deficits in world list memory, verbal fluency, and true recall as measured with the CERAD, but also with executive functions test scores as measured with CANTAB tests (Kanchanatawan et al., 2018d; Sirivichayakul et al., 2019a; 2019b). The mechanistic theory is that these different pathways have neurotoxic activities causing functional aberrations in neuronal circuits (neurogenesis, neuroplasticity, and neuronal apoptosis) underpinning the cognitome and symptomatome of schizophrenia, including "prefronto-temporal, prefronto-parietal, prefronto-striato-thalamic, and hippocampal and amygdalal neural circuits" (Orellana et al., 2013; Orellana and Slachevsky, 2013; Maes et al., 2019a; 2020a). Nevertheless, there are no data whether these biomarkers are associated with specific CANTAB test results or rather with GCoDe as indicated by a single latent trait extracted from all CANTAB tests and CERAD tests.

Hence, the current study was conducted to examine a) whether in schizophrenia aberrations in the CANTAB scores of paired association learning (PAL), rapid visual information process test (RVP), spatial working memory (SWM), intra/extradimensional set shifting (IED), emotional recognition test (ERT), and One touch Stockings of Cambridge (OTS) are manifestations of a single latent trait reflecting GCoDe; and b) whether neurotoxic pathways (OSTOX, antioxidant defenses, CCL11, and IgA to TRYCATs) are associated with a single latent trait extracted from all CATAB/CERAD tests reflecting the GCoDe.

\section{Subjects and Methods. \\ Participants}

This is a case-control study in Thai adults aged 18-65 years examining clinical and neuropsychological outcomes. We recruited schizophrenic outpatients who met the diagnostic criteria for schizophrenia of the DSM-IV-TR (and who were in a clinically stable state for at least one year) at the Department of Psychiatry, Faculty of Medicine, Chulalongkorn University, Bangkok, Thailand. The controls are healthy individuals recruited by word of mouth from the same catchment area. We excluded: 1) patients with a lifetime diagnosis of other axis-I DSM-IV / DSM-V mental disorders, including bipolar disorder, major depressive disorder, substance use disorders, and psycho-organic disorders; 2) healthy individuals with a lifetime diagnosis of any axis-I DSM-IV / DSM-V mental disorder and a family history of psychosis; and 3) healthy volunteers and schizophrenic patients with a) major medical illnesses, including rheumatoid arthritis, inflammatory bowel disease, multiple sclerosis, neurodegenerative disorders, chronic obstructive pulmonary disease, diabetes type 1 and 2 ; b) treatments with immunomodulatory drugs and antioxidant supplements and alcohol abuse; and c) pregnant and lactating women.

Primary deficit schizophrenia is defined according to criteria of the Schedule for the Deficit Syndrome (SDS) (Kirkpatrick et al., 1989): at least 2 of the following 6 features must be present with a clinically significant severity: restricted affect, diminished emotional range, poverty of speech, curbing of interest, a diminished sense of purpose, and diminished social drive, for the preceding 12 months. Additionally, these symptoms should not be secondary to for example depression or extrapyramidal side effects induced by antipsychotic medication. All participants and their guardians (parents or other close family members) gave written informed consent prior to participation in this study. The study was conducted according to Thai and international ethics and privacy laws. Approval for the study was obtained from the Institutional Review Board of the Faculty of Medicine, Chulalongkorn University, Bangkok, Thailand (No 298/57), which is in compliance with the International Guideline for Human Research protection as required by the Declaration of Helsinki, The Belmont Report, CIOMS 
Guideline and International Conference on Harmonization in Good Clinical Practice (ICHGCP).

\section{Methods}

Both healthy individuals and schizophrenia patients underwent a comprehensive clinical interview performed by a senior research psychiatrist and a trained clinical research assistant with a master's degree in mental health. We used a semi-structured interview, including the Mini-International Neuropsychiatric Interview (M.I.N.I.) in a validated Thai translation (Kittirathanapaiboon and Khamwongpin, 2005) and clinical assessments both made by the senior psychiatrist: 1) The Schedule for Deficit Syndrome (SDS) (Kirkpatrick et al., 1989); 2) The Scale for the Assessment of Negative Symptoms (SANS) (Andreasen, 1989); 3) The Positive and Negative Syndrome Scale (PANSS) (Kay et al., 1986); 4) The Brief Psychiatric Rating Scale (BPRS) (Overall and Gorham, 1962); and 5) the Hamilton Depression (HDRS) (Hamilton, 1960). We calculated z unit-weighted composite scores reflecting psychosis, hostility, excitation, mannerism, PMR, and FTD using different items of the BPRS, HDRS, and PANNS (Maes et al., 2019claudin). Electronic Supplementary File 1 (ESF 1), Table 1 shows the items that were employed to compute these symptom subdomain scores. HR-QoL was assessed with the World Health Organization Quality of Life Instrument-Abbreviated version (WHO-QoL-BREF) (WHO, 1993), which measures four domains: 1) Domain 1 or physical health; 2) Domain 2 or psychological health; Domain 3 or social relationships; and 4) Domain 4 or environment. The raw scores were computed according to WHO-QoL-BREF criteria (WHO, 1993). We also assessed tobacco use disorder (TUD) according to DSM-IV-TR criteria.

\section{Cognitive assessments}

Nine key Outcome Measures for CANTAB Research Suite Tests were used as advocated by CANTAB (CANTAB 2020) to evaluate neurocognitive functions:

a) Paired-association learning (PAL) to assess visual memory, episodic memory, and learning. We analyzed PAL total errors adjusted (PAL-TEA), "the number of times the subject chose the incorrect box for a stimulus on assessment problems but with an adjustment for the estimated number of errors they would have made on any problems, attempts \& recalls they did not reach due to failing or aborting the test".

b) Rapid visual information process test (RVP) to assess visual sustained attention. We used RVP A' Prime (RVP_A), "the signal detection measure of sensitivity to the target, regardless of response tendency (the expected range is 0.00 to 1.00 ; bad to good). In essence, this metric is a measure of how good the subject is at detecting target sequences"; and RVP median latency (RVP_ML), "the median response latency during assessment sequence blocks where the subject responded correctly".

c) Spatial working memory (SWM) to assess working memory and strategy use. We analyzed SWM between errors (SWM-BE), "the total number of times the subject revisits a box in which a token has previously been found in the same problem"; and and SWM strategy (SWM-STR), for assessed problems with six boxes or more, the number of distinct boxes used by the subject to begin a new search for a token, within the same problem;

d) One touch stockings of Cambridge (OTS) to assess spatial planning. We analyzed OTS probability solved on first choice (OTS-PSOFC), "the number of assessment problems on which the first box choice made was correct".

e) Intra/extradimensional set shifting (IED) to assess rule acquisition and attention setshifting. We measured IED EDS errors (IED_EDS), "the number of times that the subject failed to select the stimulus compatible with the current rule on the stage where the ED dimension shift occurs"; and IED total errors adjusted (IED-TEA), "the total number of times 
that the subject chose a stimulus incompatible with the current rule, plus, for each problem that was not reached (if any), an adjustment is made to the score";

f) Emotional recognition test (ERT), an emotional recognition task to recognize facial expression of emotion. We analyzed 2 ERT outcome measures, i.e. ERT mean overall response latency (ERT-MORL), "the median latency from stimulus onset to the subject's response button touch (i.e. emotion chosen) for all problems during assessment blocks. ESF 2, Methods, Table 1 lists all CANTAB tests that were performed in the subjects.

We also measured three CERAD tests, namely the Word List Memory (WLM) to assess verbal episodic memory, the Verbal Fluency Test (VFT) to assess semantic memory, and Word List True recall to assess verbal episodic memory recall. Finally, we also measured the Mini Mental State Examination (MMSE) to assess a more generalized cognitive deficit including in ideatoric and constructional praxis, orientation, speech, concentration, and memory.

Assays.

Blood was sampled at 8.00 a.m. after an overnight fast and thawed for assay. All assays were conducted in one and the same run by the same operator who was blinded to the clinical results. As described previously (Kanchanatawan et al., 2018), ELISA tests were employed to assay the plasma titers of immunoglobulins (Ig) A (IgA) directed against the tryptophan catabolites xanthurenic acid (XA) (Acros), picolinic acid (PA) (Acros), 3-OHkynurenine (3HK) (Sigma), kynurenic acid (KA) (Acros), and anthranilic acid (AA) (Acros), linked to $20 \mathrm{mg}$ BSA (ID Bio). Optical densities (ODs) were measured at $450 \mathrm{~nm}$ using Varioskan Flash (Thermo Scientific). Consequently, we computed the noxious / generally more protective (NOX/PRO) TRYCAT ratio as shown in ESF, Table 1. Serum CCL11 (R\&D Systems, Inc, Minneapolis, MN, USA) was measured using the Bio-Plex ${ }^{\circledR} 200$ System (BioRad Laboratories, Inc.) as reported previously (Sirivichayakul et al., 2019b). The methods for the OSTOX and ANTIOX assays were described previously (Roomruangwong et al., 2020; Maes et al., 2020c): "MDA levels were measured through complexation with two molecules of thiobarbituric acid using MDA estimation through high-performance liquid chromatography (HPLC Alliance e2695, Waters', Barueri, SP, Brasil). AOPP was quantified in a microplate reader (EnSpire, Perkin Elmer, USA) at a wavelength of $340 \mathrm{~nm}$ and is expressed in $\mathrm{mM}$ of equivalent chloramine $\mathrm{T}$. LOOH was quantified by chemiluminescence in a Glomax Luminometer (TD 20/20), in the dark, at $30{ }^{\circ} \mathrm{C}$ for $60 \mathrm{~min}$ and the results are expressed in relative light units. TRAP was evaluated in a microplate reader (Victor X-3, Perkin Elmer, USA) and results are expressed in $\mu \mathrm{M}$ Trolox. -SH groups were evaluated in a microplate reader (EnSpire ${ }^{\circledR}$, Perkin Elmer, USA) at a wavelength of $412 \mathrm{~nm}$ and results are expressed in $\mu \mathrm{M}$. The substrates used to assess PON1 activity were phenyl acetate (PA, Sigma, USA) under high salt condition and CMPA (Sigma, USA), which is an alternative to the use of the toxic paraoxon. PON1 activities were determined by the rate of hydrolysis of CMPA (CMPAase) as well as by the rate hydrolysis of phenyl acetate under low salt condition. Analysis were conducted in a microplate reader (EnSpire, Perkin Elmer, USA). Consequently, we computed a $\mathrm{z}$ unit-weighted composite score reflecting the OSTOX/ANTIOX ratio as explained in ESF, Table 1.

\section{Statistics}

We employed analysis of contingency tables $\left(\mathrm{X}^{2}\right.$-test) to check associations between categorical variables and analysis of variance (ANOVA) or the Mann-Whitey-U test to assess differences in scale or ordinal variables between diagnostic classes (controls and schizophrenia with and without the deficit syndrome). We employed multivariate general linear model (GLM) analysis to assess the association between diagnostic groups and a set of 
CANTAB variables while adjusting for age, sex, education, and other background variables. When significant, we used tests for between-subject effects to check the associations between diagnosis and each of the CANTAB data. Results of multiple comparisons were always corrected for false discovery rate (FDR) (Benjamini and Hochberg, 1995). Subsequently, we computed model-generated estimated marginal mean (SE) values and employed protected, pairwise LSD tests to assess differences between the three diagnostic groups. Automatic (step-up) binary logistic regression analysis was employed to delineate the most important predictors of schizophrenia (versus controls) or deficit schizophrenia (versus non-deficit schizophrenia) using cognitive test results as discriminating variables. Power analysis showed that for an ANCOVA with three categories and five covariates, an effect size $\mathrm{f}=0.30$, power $=0.8$, and $\alpha=0.05$, the required sample size should be around $n=111$. Given a possible dropout rate of $8 \%$ we recruited 120 participants. Statistical analyses were performed using IBM SPSS Windows version 25. Tests were 2-tailed, and an alpha level of 0.05 indicated a statistically significant effect.

Exploratory factor analysis (EFA), FACTOR, windows version 10.5.03 (Ferrando and Lorenzo-Seva, 2017; Lorenzo-Seva and Ferrando, 2019) was employed to explore the factor structure of the neurocognitive tests scores. The dispersion matrix used Pearson's correlations and we extracted factors with the robust unweighted least squares (RULS) method performed with 500 bias-corrected and accelerated (BCa) bootstraps (Ferrando and Lorenzo-Seva, 2017; Lorenzo-Seva and Ferrando, 2019). The adequacy for factorization was checked with the Kaiser-Meyer-Olkin (KMO) test and Bartlett's test of sphericity. To estimate the number of factors to be retained we employed the Hull test and Parallel Analysis (Optimal Implementation) and Schwartz's Bayesian Information Criterion (BIC). We checked closeness to unidimensionality with explained common variance (ECV), unidimensional congruence (UNICO), and the mean of item residual absolute loadings (MIREAL). Data may be treated as essentially unidimensional when ECV $>0.85$, UNICO $>0.95$, and MIREAL $<0.300$. In order to check the model goodness-of-fit we employed the goodness-of-fit index (GFI). We assessed the distribution of residuals with the Weighted Root Mean Square Residual (WRMR) whereby values $<1.0$ indicate an adequate fit. We assessed construct replicability with the Generalized $\mathrm{H}$ index with values $\geq 0.80$ indicating good stability across studies.

Subsequently, we conducted Partial Least Squares (PLS) path analysis (SmartPLS) (Ringle et al., 2014), which was used to examine the associations between a latent vector (LV) extracted from the 9 key CANTAB, MMSE, VFT, WLM, and True Recall scores (reflecting GCoDe) in a reflective model, and the biomarkers (entered as single indicators), the symptomatome (a LV extracted from PHEMN symptoms, FTD and PMR) and the phenomenome (a LV extracted from the 4 WHO-QoL domains). The three single indicators, namely the OSTOX/ANTIOX ratio, CCL11, and IgA NOX/PRO, predicted the cognitome, symptomatome, and phenomenome. We conducted complete PLS path analysis only when the constructs and the model fit complied with pre-specified quality criteria, namely: a) all factor loadings are significant $(\mathrm{p}<0.001)$ and $>0.500$ (by preference 0.660 ); $b$ ) the LVs show good construct validity or internal consistency reliability and convergent validity as indicated by Cronbach alpha $>0.750$, composite reliability $>0.800$, rho_A $>0.800$, and average variance extracted $(\mathrm{AVE})>0.500$; c) the model fit is adequate as assessed with the standardized root mean residual (SRMR) $<0.08$; and $\mathrm{d}$ ) the cross-validated predictive relevance of the PLS path model is adequate as assessed with the cross-validated redundancy approach with blindfolding, a predictive sample re-use technique. The latter was performed with an omission distance of 7 to compute the Stone-Geisser's $\mathrm{Q}^{2}$ statistic whereby values of $\mathrm{Q}^{2}>0.02,0.15$, and 0.35 indicate that the model has a small, medium, and large predictive relevance for the selected construct. Indicators of the inner and outer model or constructs that 
do not comply with those quality data are eliminated from the final model. Subsequently, complete PLS bootstrapping (with 5000 bootstrap samples) is performed with calculation of the t-values and loadings of the indicators in the outer model, and the path coefficients for the inner model. Total, total indirect, and specific indirect effects are calculated. Confirmatory Tetrad Analysis (CTA) is conducted to assess possible misspecifications of a reflective LV model.

\section{Results.}

Socio-demographic and clinical data

Table 1 shows the demographic and clinical data for the three study groups. There were no significant differences in age and education between the three groups. In the schizophrenic study groups, there were significantly more men and single individuals without work than in the control group. There was no significant difference in duration of illness or number of psychotic episodes between deficit and nondeficit schizophrenia. BMI was significantly lower in patients with deficit schizophrenia than in those with nondeficit schizophrenia. Only seven people showed TUD. This table also shows the measurements of the three CERAD tests including MMSE and shows the estimated marginal means after adjusting for age, sex, and education years. The MMSE and True Recall scores were significantly lower in deficit schizophrenia than in the two other groups. VFT and WLM were significantly different between the three study groups.

ESF 1, Figure 1 shows that all symptom domain scores (except hostility) were significantly different between the three study groups and increased from controls to nondeficit and deficit schizophrenia. ESF 1, Figure 2 shows that the WHO-Qol domain scores were significantly different between the study groups and decreased from controls to nondeficit and deficit schizophrenia (except domain 3).

\section{CANTAB measurements in schizophrenia}

Table 2 shows the outcome of a multivariate GLM analysis which examined the associations between the diagnosis and the 9 CANTAB test results, while adjusting for age, education, and gender. We found a highly significant association between the diagnosis and the 9 CANTAB test results and significant effects of age and education, but not gender. Diagnosis shared around $23.1 \%$ of the variance with the CANTAB data. Univariate GLM analyses showed that there were significant associations between diagnosis and all CANTAB variables except IED_EDS and IED_TEA. False discovery rate correction did not change these results. Table 3 shows the estimated marginal mean values of the 9 CANTAB measures in the three study groups. We found that participants with deficit schizophrenia showed worse outcomes on all measurements than controls (except IED_EDS). Moreover, PAL_TEA, RVP_A and ERT_MORL were significantly different between participants with deficit and nondeficit schizophrenia.

ESF 1, Section 1 describes the effects of age, sex, and education on the CANTAB measurements. This section also describes that tobacco use disorder, BMI, employment, and the drug status did not have any significant effects on the CANTAB test results.

\section{Results of exploratory factor analysis}

Table 4 shows the results of an exploratory factor analysis with the CANTAB tests combined with the three CERAD tests and MMSE scores as variables. A first analysis showed that all variables had loadings $>0.500$, except IED_EDS (0.103) and, therefore, we have eliminated the latter CANTAB test score from the final analysis shown in Table 4 . The KMO statistic of sampling adequacy was 0.863 and Bartlett's test $(\chi 2=810.0$, df $=66$, $\mathrm{p}<0.00001$ ) show adequate factorability of the correlation matrix. We found that one real-data 
eigenvalue was greater than 1.0, and that the first factor explained $50.5 \%$ of the variance in the 12 cognitive scores. The BIC and Hull test, and PA analysis indicated that the advised number of factors was one. Moreover, the ECV $(\geq 0.850)$, UNICO $(\geq 0.95)$, and MIREAL $(\leq 0.3)$ values indicated that the first factor extracted from the 12 cognitive tests should be treated as unidimensional. Table 4 shows that all cognitive scores loaded highly $(>0.500)$ and that the model goodness of fit index showed adequate values. In addition, also the distribution of residuals as assessed with weight root mean square residuals showed a good fit. Finally, the Generalized H index of 0.923 indicated an adequate construct replicability and performance across studies.

\section{Examination of all CANTAB tests assessed in this study}

ESF 2, Methods, Table 1 shows all CANTAB tests that we measured in this study. ESF 2, Statistics describes how we extracted principal components (PC) using SPSS 25 from all CANTAB tests in the 7 CANTAB subdomains. ESF 2, Results, Tables 1 and 2 show the results of multivariate GLM analyses and consequent tests for between-subject effects performed on the PCs extracted from the 7 CANTAB subdomains. The results show that nondeficit patients have significant impairments in PC PAL, PC SWM, PC OTS, PC IED, and PC ERT as compared with healthy controls. Deficit schizophrenia was characterized by significant impairments in PC RVP, which were not present in nondeficit schizophrenia, and significantly more dysfunctions in PC PAL, PC SWM and PC ERT, as compared with nondeficit schizophrenia.

ESF 2, Results, Tables 3-5 show all the CANTAB tests that were measured in this study in the three study groups. ESF 2, Results, Table 6 shows the results of binary logistic regression analyses discriminating schizophrenia from controls and deficit from non-deficit schizophrenia.

\section{Results of PLS analysis}

Figure 1 shows the results of a first PLS analysis with a LV extracted from the four HR-QoL subdomain scores as final output variable, and with the symptomatome (introduced as a latent vector extracted from PHEMN symptoms, PMR, and FTD) and the cognitome (latent vector extracted from the key CANTAB tests, MMSE, VFT, WLM, and True Recall) as direct input variables, which may mediate the effects of the biomarkers which were entered as single indicators, namely IgA NOX/PRO TRYCATs, CCL11, and the OSTOX/ANTIOX ratio. The model fit was more than adequate with a SRMR of 0.052 for the saturated model and 0.054 for the estimated model. The construct validities of all latent vectors were adequate with Cronbach $\alpha>0.893$, composite reliabilities $>0.923$, rho_A $>$ 0.911 , and AVEs $>0.523$. The loadings on all LVs were $>0.500$ at $p<0.001$. The cognitome LV showed a Cronbach $\alpha$ of 0.907 and an AVE of 0.523 with all loadings $>0.651$, except ERT_MORL (0.564). PLS path analysis conducted with 5000 bootstraps showed that $40.9 \%$ of the variance in the phenomenome LV was explained by the symptomatome and cognitome $\mathrm{LV} ; 47.4 \%$ of the variance in the symptomatome LV was explained by the cognitome and IgA TRYCATs; and $40.5 \%$ of the variance in the cognitome was explained by the cumulative effects of the OSTOX/ANTIOX ratio, IgA TRYCATS, and CCL11. We found that all the mediated effects of the three biomarkers on the symptomatome and phenomenome were significant. The cognitome (see Figure 1) has significant direct and specific indirect effects on the phenomenome mediated by the symptomatome $(\mathrm{t}=4.06, \mathrm{p}<0.001)$. Blindfolding showed that the replicability of the cognitome LV was adequate, namely cross-validated redundancy $=0.203$. Multi-group analysis showed that there were no significant differences between both sexes in the paths, indirect and direct effects. Confirmatory Tetrad Analysis showed that the cognitome LV was not misspecified as a reflective model. 
Consequently, we have performed a second PLS analysis which is quite similar to the analysis shown in Figure 1 except that we used some other indicators for the cognitome LV, namely the first PCs extracted from all IED, OTS, PAL, RVP, SWM, and ERT tests, and in addition the three CERAD tests including MMSE. The model fit was more than adequate with a SRMR of 0.050 for the saturated model and 0.053 for the estimated model. The construct validity of the cognitome LV was very good with Cronbach $\alpha=0.921$, composite reliability $=0.934$, rho_A $=0.924$, and AVEs $>0.586$. Figure 2 shows that the loadings on this LV were $>0.702$ at $p<0.001$. Figure 2 shows that $39.9 \%$ of the variance in the cognitome was explained by the three biomarkers. We found that the three biomarkers had significant effects on the symptomatome and the phenomenome, which were mediated by the cognitome. The latter showed significant direct (see Figure 2) and specific indirect $(t=4.31, p<0.001)$ effects on the phenomenome, and that the latter were mediated by the symptomatome.

Consequently, we have computed the latent variable scores for the three CERAD scores (3CERAD), the three CERAD scores and the MMSE score (4CERAD), the 9 key CANTAB scores (9CANTAB), the 4 CERAD +9 key CANTAB scores (CERAD+CANTAB), and the 7 PCs of the 7 CANTAB subdomains (PC7CANTAB). Figure 3 shows a clustered bar graph of these 5 values in controls and schizophrenia patients with and without deficit syndrome. Univariate GLM analysis with age, sex, and education as covariates showed significant intergroup differences in the 3CERAD $(\mathrm{F}=29.54, \mathrm{df}=2 / 110$, $\mathrm{p}<0.001$, partial eta squared $=0.349), 4$ CERAD $(\mathrm{F}=29.05, \mathrm{df}=2 / 110, \mathrm{p}<0.001$, partial eta squared $=0.346), 9$ CANTAB $(\mathrm{F}=28.05, \mathrm{df}=2 / 110, \mathrm{p}<0.001$, partial eta squared $=0.285)$, CERAD + CANTAB $(\mathrm{F}=31.42, \quad \mathrm{df}=2 / 110, \quad \mathrm{p}<0.001, \quad$ partial eta squared $=0.364)$, and PC7CANTAB $(\mathrm{F}=16.39, \mathrm{df}=2 / 110, \mathrm{p}<0.001$, partial eta squared $=0.230)$. All scores were significantly different between the three groups and increased from controls $\rightarrow$ nondeficit $\rightarrow$ deficit schizophrenia. There was a strong association between CERAD+CANTAB and 3 CERAD $(\mathrm{r}=0.834, \mathrm{p}<0.001, \mathrm{n}=116)$.

Discussion A generalized cognitive decline (GCoDe) in schizophrenia

The first major finding of this study is that, in schizophrenia, a single latent trait, which is essentially unidimensional, underpins the 9 key CANTAB, MMSE, VFT, WLM, and True Recall scores. Moreover, this latent construct fitted a reflective model and showed adequate psychometric properties indicating good convergent validity, excellent composite reliability, and adequate construct replicability. As such, the latent phenomenon GCoDe is reflectively measured through its 13 effect indicators, which are, therefore, to a large extent modulated by the GCoDe. These findings indicate that, in schizophrenia, the aberrations in visual sustained attention, working memory and strategy use, rule acquisition and attention set-shifting, emotional recognition, semantic memory, episodic memory, and episodic memory recall are strongly interrelated. As such, the GCoDe reflects aberrations in neuronal circuits that underpin these CANTAB/CERAD tests including prefronto-temporal, prefrontoparietal, hippocampal, and fronto-striatal neural circuits (Orellana et al., 2013; Orellana and Slachevsky, 2013; Maes et al., 2019b; 2020a).

\section{The GCoDe in nondeficit schizophrenia}

The second major finding of this study is that nondeficit schizophrenia is characterized by significantly deficits in visual memory, semantic memory, episodic memory recall, episodic memory and learning, spatial working memory and strategy use, spatial planning, rule acquisition and attention set-shifting, and interpretation of facial expression of emotion. As such, nondeficit schizophrenia is characterized by a multiform cognitive decline (namely the GCoDe), which is the common cause of the cognitive manifestations as assessed 
with CANTAB and CERAD tests. Previously, Levaux et al. (2007) reviewed the CANTAB findings in schizophrenia and showed widespread cognitive impairments in working memory, decision-making, attention, visual memory, and especially in attentional set-shifting. Deficits in episodic memory, long-term memory, and conditional associative learning tasks are now well documented in schizophrenia (Rushe et al., 1999; Leavitt and Goldberg, 2009). Our findings on impaired visual memory in schizophrenia agree with those of Seidman et al. (2003) and Kim et al. (2008) who observed visual memory disorders in schizophrenia.

Moreover, our study also showed an overall decline in executive functions in schizophrenia, which corresponds to the findings of Levaux et al. (2007) and Pantelis et al. (1997). The impaired SWM performance in schizophrenia suggests impaired working memory and strategy use, which extends previous findings (Zilles et al., 2010; Pantelis et al., 2001; Stone et al., 1998; Badcock et al. 2005). Importantly, Bonner-Jackson et al. (2008) reported that a deficit in the use of effective strategies in schizophrenia may modulate episodic memory performance. The current study showed significantly poorer IED scores in schizophrenia patients, suggesting disordered rule acquisition and attention set-shifting, while no such results were described by Hilti et al. (2010). Our OTS findings in schizophrenia patients indicate impairments in spatial planning, which agrees with previous reports (Fraser et al. 2004; Badcock et al., 2005; Hilti et al., 2010). Nevertheless, our factor analysis shows that the results on cognitive functioning in schizophrenia should not be discussed on a testby-test basis, but rather by the new knowledge that all these test scores are reflective manifestations of a GCoDe.

\section{The GCoDe in deficit schizophrenia}

The third major finding of this study is that the severity of the GCoDe, different key CANTAB tests, most PCs extracted from the subdomain tests scores, and many tests from the different CANTAB domains, and VFT, WLM, True Recall, and MMSE scores were significantly more pronounced in deficit than in nondeficit schizophrenia. Previous studies in deficit schizophrenia showed somewhat inconsistent results with significant or no significant changes in deficit versus nondeficit schizophrenia (review: Yu et al., 2015). The latter review detected more impairments in general cognitive capacities and executive functions, sustained attention, and visuospatial memory in deficit than in nondeficit schizophrenia ( $\mathrm{Yu}$ et al., 2015). Yu et al (2015) concluded that impairments in cognitive flexibility and sustained attention are the most important dysfunctions in deficit schizophrenia. But again, such deductions are less than optimal as deficit schizophrenia is characterized by increased severity of the latent phenomenon GCoDe, which underpins the many cognitive manifestations as assessed with CANTAB/CERAD.

Interestingly, there was a trend in reaction times and latency measures to be greater in deficit schizophrenia for RVP (more impaired in deficit than in nondeficit schizophrenia), OTS, and SWM (significantly greater impairment in deficit schizophrenia than in controls, whilst nondeficit schizophrenia patients have an intermediate position) and IED and ERT (significantly greater latency in deficit schizophrenia compared to nondeficit schizophrenia). This is particularly interesting in ERT, where ERT-MORL is a measure to differentiate between deficit and nondeficit schizophrenia. The same can be said for IED-total latency. Such findings suggest a slower processing in deficit schizophrenia.

GCoDe versus specificity of the cognitive CANTAB tests

Based on our findings that a common single trait underpins all CANTAB/CERAD tests, we may conclude that it is not adequate to claim that the key CANTAB tests are specific tests, which reflect aberrations in distinct functions in schizophrenia (CANTAB, 
2020). If we would have employed only the key CANTAB tests we would have concluded that deficit schizophrenia is characterized by highly specific dysfunctions in pairedassociation learning, rapid visual processing, and interpretation of facial expression of emotion, whereas in fact schizophrenia is characterized by a GCoDe.

Moreover, some tests, which are not considered to be key tests by CANTAB (2020) are more relevant for deficit schizophrenia than the key tests of the same domain. For example, PAL_FTMS, IED_TE, IED_TT, RVP_TCR, RVP_TM, and RVP_TH are more significantly associated with deficit schizophrenia than the key tests in the same domain, namely PAL_TEA, IED_TEA, and RVP_A. In the discrimination of deficit from nondeficit schizophrenia we found that PAL_SCFT, which is not a key test according to CANTAB, has more discriminatory power than the PAL key tests. When discriminating schizophrenia from controls, SWM_IED is a more significant discriminatory test than the key CANTAB tests. Furthermore, using the first PC extracted from all tests in the subdomains allowed us to detect additional aberrations in the SWM and IED domains in deficit schizophrenia.

Last but not least, the CERAD tests have more significant discriminatory power than the CANTAB tests. For example, VFT and WLM test scores are more important than SWM_IED and ERT_MORL in discriminating schizophrenia from controls, while lowered True Recall is the single best predictor of deficit from non-deficit schizophrenia. Moreover, the use of the CANTAB is hampered by the strong effects of education and age which explain a large part of the variability in the 9 key CANTAB tests, namely $35.2 \%$ and $26.7 \%$, respectively. The WLM and True Recall tests results are much less prone to the effects of education and are not affected by age (Kanchanatawan et al., 2018a). The three CERAD tests, VFT, WLM and True Recall, are somewhat more adequate to measure cognitive deficits in schizophrenia than the 9 key CANTAB tests. Consequently, we would propose that a $\mathrm{z}$ unit composite score based on three CERAD tests, namely VFT, WLM, and True Recall, may be used as a convenient proxy for the GCoDe in schizophrenia.

\section{GCoDe mediates the effects of pathways on the phenome of schizophrenia}

The fourth major finding of this study is that the severity of the GCoDe in schizophrenia is strongly predicted by neurotoxic pathways and mediates in part the effects of those pathways on the symptomatome (PHEMN, PMR, and FTD) and phenomenome (lowered HR-Qol scores). The severity of GCoDe was to a large extent (40.2\%) explained by increased oxidative stress toxicity and lowered antioxidant defenses (the OSTOX/ANTIOX ratio), CCL11, a neurotoxic chemokine that impacts neurogenesis, and increased activity of the TRYCAT pathway with increased IgA levels to the NOX/PRO ratio (Kanchanatawan et al., 2018a; Sirivichayakul et al., 2019a; 2019b; Maes et al., 2020b). These results indicate that the neuro-immune and neuro-oxidative pathways impact GCoDe through a multitude of neurotoxic effects including on neuroplasticity, synapse functions, protein regulation processes, cell signaling, apoptosis, and transcription (Maes et al., 2020b; 2020c). As explained previously, these neurocognitive impairments may be causally associated with the PHEMN symptoms, FTD, and PMR of schizophrenia, and consequently with lowered HRQoL (Sirivichayakul et al., 2019a; Maes et al., 2020a; 2020b; 2000c; Kanchanatawan et al., 2018a). Cognitive deficits are viewed as a direct result of "compromised cerebral function" (Tamminga et al., 1998) preceding psychotic symptoms (Harvey et al., 2006; Tamminga et al., 1998).

It is heavily debated whether impairments in specific cognitive tests are associated with positive or negative symptoms (Bombin et al., 2013; Sewell et al., 2010; Saleem et al., 2013; Eack et al., 2013; Sanchez et al., 2014; O’Leary et al., 2000). For example, Lin et al. (2013) found that negative symptoms may mediate the effects of cognition on functional outcomes. Yu et al. (2015) reported significant correlations between negative symptoms and 
sustained attention, ideation fluency, cognitive flexibility, and visuospatial memory. Harvey (2006) stated that the true nature of the associations between negative symptoms and cognitive impairments are still elusive. However, these discussions are not relevant, because PHEMN symptoms, FTD, and PMR are reflective manifestations of a generalized underlying trait, namely OSOS (Almulla et al., 2020; Maes et al., 2019a). Furthermore, the current study reported that GCoDe, a single latent trait underlying all neurocognitive impairments, explained a large part (44.8\%) of the variance in OSOS, the latent phenomenon underpinning all symptom domains of schizophrenia.

\section{Limitations}

The results of the current study should be interpreted with regard to its limitations. First, this is a case-control study, which does not allow to draw solid conclusions on causal models. Second, it would have been more interesting if we had performed functional neuroimaging techniques including multimodal imaging and imaging immunology (Stoyanov et al., 2017) to detect which neuronal circuits are associated with the GCoDe.

\section{Conclusions}

In schizophrenia, a single latent phenomenon underlies various CANTAB and CERAD test scores of semantic and episodic memory, recall, executive functions, strategy use, rule acquisition, visual sustained attention, attention set-shifting, and emotional recognition. This latent phenomenon fits a reflective model and indicates that a GCoDe is the cause of its cognitive manifestations as assessed with single CANTAB and CERAD tests. Patients with deficit schizophrenia show a more profound GCoDe than those with nondeficit schizophrenia. GCoDe mediates the neurotoxic effects of immune and oxidative pathways on the symptomatome and phenomenome of schizophrenia. 
Table 1. Demographic and clinical data in normal controls and schizophrenia patient with and without deficit syndrome.

\begin{tabular}{|c|c|c|c|c|c|c|}
\hline Variables & Normal Control $^{\mathbf{A}}$ & Nondeficit SCZ ${ }^{B}$ & Deficit SCZ $^{\text {C }}$ & $F / X^{2}$ & df & $\mathbf{p}$ \\
\hline Age (years) & $37.4(12.8)$ & $41.3(10.8)$ & $40.9(11.4)$ & $2 / 117$ & $2 / 117$ & 0.259 \\
\hline Gender $(\mathrm{M} / \mathrm{F})$ & $10 / 30^{\mathrm{BC}}$ & $22 / 18^{\mathrm{A}}$ & $21 / 19^{\mathrm{A}}$ & 8.99 & 2 & 0.011 \\
\hline Education (years) & $14.2(4.9)$ & $12.8(4.2)$ & $11.9(4.1)$ & 3.03 & $2 / 117$ & 0.052 \\
\hline Single-divorced/Married & $26 / 14^{\mathrm{C}}$ & $31 / 8$ & $36 / 3^{\mathrm{A}}$ & 8.84 & 2 & 0.012 \\
\hline Work status $(\mathrm{N} / \mathrm{Y})$ & $4 / 36^{\mathrm{B}, \mathrm{C}}$ & $16 / 29^{\mathrm{A}, \mathrm{C}}$ & $30 / 10^{\mathrm{A}, \mathrm{B}}$ & 34.83 & 2 & $<0.001$ \\
\hline Duration of illness (years) & - & $15.7(10.1)$ & $15.1(12.3)$ & MWU & - & 0.585 \\
\hline Number of psychotic episodes & - & $2.95(2.0)$ & $3.43(3.37)$ & MWU & - & 0.791 \\
\hline $\operatorname{BMI}\left(\mathrm{kg} / \mathrm{m}^{2}\right)$ & $24.0(4.3)$ & $26.0(5.2)^{\mathrm{C}}$ & $22.9(4.6)^{\mathrm{B}}$ & 4.40 & $2 / 112$ & 0.005 \\
\hline TUD $(\mathrm{Y} / \mathrm{N})$ & $2 / 38$ & $4 / 36$ & $1 / 39$ & NA & - & - \\
\hline MMSE* & $27.59(0.42)^{\mathrm{C}}$ & $26.80(0.40)^{\mathrm{C}}$ & $25.42(0.40)^{\mathrm{A}, \mathrm{B}}$ & 7.07 & $2 / 114$ & 0.001 \\
\hline VFT* & $26.39(1.00)^{\mathrm{B}, \mathrm{C}}$ & $20.16(0.91)^{\mathrm{A}, \mathrm{C}}$ & $17.02(0.91)^{\mathrm{A}, \mathrm{B}}$ & 25.00 & $2 / 114$ & $<0.001$ \\
\hline WLM* & $20.95(0.70)^{\mathrm{B}, \mathrm{C}}$ & $18.75(0.67)^{\mathrm{A}, \mathrm{C}}$ & $15.59(0.67)^{\mathrm{A}, \mathrm{B}}$ & 15.12 & $2 / 114$ & $<0.001$ \\
\hline True Recall & $7.67(0.30)^{\mathrm{C}}$ & $7.26(0.29)^{\mathrm{C}}$ & $5.46(0.29)^{\mathrm{A}, \mathrm{B}}$ & 16.09 & $2 / 114$ & $<0.001$ \\
\hline
\end{tabular}

$\mathrm{F} / \mathrm{X}^{2}$ : results of analyses of variance $(\mathrm{F})$ or analyses of contingency analyses $\left(\mathrm{X}^{2}\right)$. Results are shown as mean $(\mathrm{SD})$, except $*$ : estimated marginal means $(\mathrm{SE})$ obtained by GLM analysis after covarying for sex, age, and education.

A,B,C: pairwise comparisons among treatment means. ${ }^{\text {A: }}$ : significantly different from controls, ${ }^{\text {B: }}$ from nondeficit schizophrenia, ${ }^{\text {: }}$ from deficit schizophrenia (protected post-hoc tests)

BMI: body mass index; TUD: tobacco use disorder; MMSE: Mini Mental State Examination; VFT: Verbal Fluency Test; WLM: World List memory 
Table 2. Results of multivariate GLM analyses with the 10 CANTAB data as dependent variables and diagnosis as primary explanatory variable

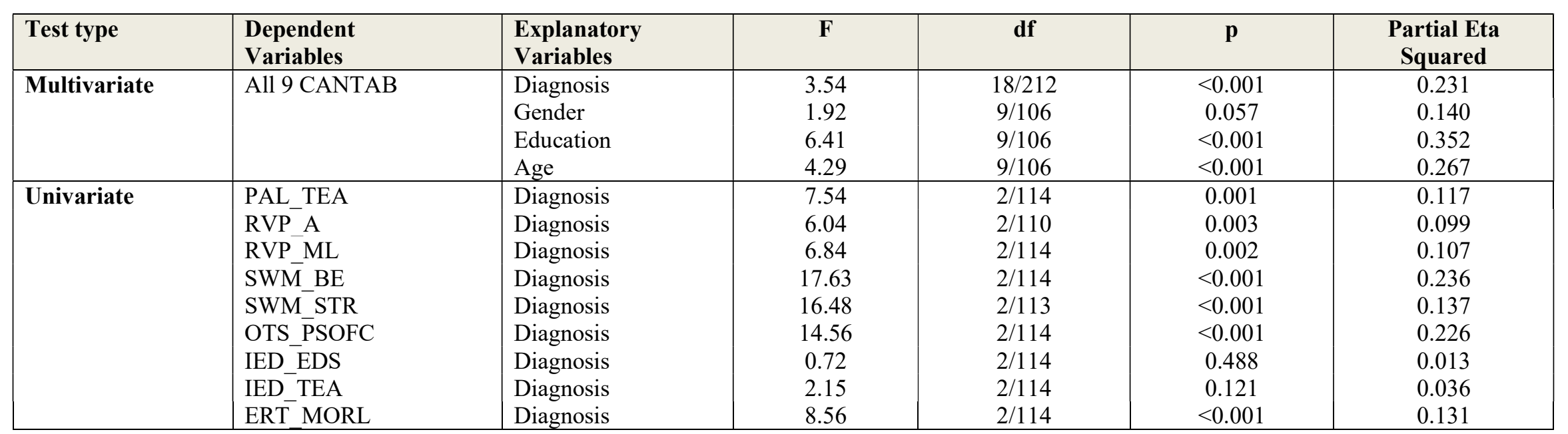

Diagnosis: three groups, namely healthy controls and patients with and without deficit schizophrenia

PAL_TEA: Paired-association learning, total errors adjusted; RVP_A: Rapid visual information process test, A' prime; RVP_ML: Rapid visual information process test, median latency; SWM_BE: Spatial working memory, between errors; SWM_STR: Spatial working memory, strategy; OTS_PSOFC: One touch stockings of Cambridge, problems solved on first choice; IED_EDS: Intra/extradimensional set shifting, shift errors; IED_TEA: Intra/extradimensional set shifting, total errors adjusted; ERT_MORL: Emotional recognition test, median overall response latency 
Table 3. Model-generated estimated marginal means (SE) obtained by univariate GLM analysis (age, sex and education adjusted)

\begin{tabular}{|l|c|c|c|}
\hline CANTAB Variables & Normal Controls $^{\mathbf{A}}$ & Nondeficit SCZ $^{\mathbf{B}}$ & Deficit SCZ $^{\mathbf{C}}$ \\
\hline PAL_TEA & $33.3(8.4)^{\mathrm{C}}$ & $46.2(7.9)^{\mathrm{C}}$ & $77.0(8.0)^{\mathrm{A}, \mathrm{B}}$ \\
\hline RVP_A & $0.963(0.010)^{\mathrm{C}}$ & $0.9480 .009^{\mathrm{C}}$ & $0.9170 .009^{\mathrm{A}, \mathrm{B}}$ \\
\hline RVP_ML & $377(27)^{\mathrm{B}, \mathrm{C}}$ & $484(26)^{\mathrm{A}}$ & $513(26)^{\mathrm{A}}$ \\
\hline SWM_BE & $30.4(3.4)^{\mathrm{B}, \mathrm{C}}$ & $50.9(3.2)^{\mathrm{A}}$ & $57.6(3.2)^{\mathrm{A}}$ \\
\hline SWM_STR & $34.2(0.8)^{\mathrm{B}, \mathrm{C}}$ & $38.7(0.8)^{\mathrm{A}}$ & $40.7(0.8)^{\mathrm{A}}$ \\
\hline OTS_PSOFC & $8.27(0.46)^{\mathrm{B}, \mathrm{C}}$ & $5.94(0.44)^{\mathrm{A}}$ & $4.80(0.44)^{\mathrm{A}}$ \\
\hline IED_EDS & $15.3(2.0)$ & $18.0(1.9)$ & $18.6(2.0)$ \\
\hline IED_TEA & $52.8(7.4)^{\mathrm{C}}$ & $60.5(7.0)$ & $73.8(7.0)^{\mathrm{A}}$ \\
\hline ERT_MORL & $2749(248)^{\mathrm{B}, \mathrm{C}}$ & $3525(235)^{\mathrm{A}, \mathrm{C}}$ & $4191(237)^{\mathrm{A}, \mathrm{B}}$ \\
\hline
\end{tabular}

A,B,C: pairwise comparisons among treatment means. ${ }^{A}$ : significantly different from controls, ${ }^{B}$ from nondeficit schizophrenia, ${ }^{\mathrm{C}:}$ from deficit schizophrenia (protected post-hoc tests)

PAL_TEA: Paired-association learning, total errors adjusted; RVP_A: Rapid visual information process test, A' prime; RVP_ML: Rapid visual information process test, median latency; SWM_BE: Spatial working memory, between errors; SWM_STR: Spatial working memory, strategy; OTS_PSOFC: One touch stockings of Cambridge, problems solved on first choice; IED_EDS: Intra/extradimensional set shifting, shift errors; IED_TEA: Intra/extradimensional set shifting, total errors adjusted; ERT_MORL: Emotional recognition test, median overall response latency 
Table 4. Results of exploratory factor analysis performed on key CANTAB and CERAD tests.

\begin{tabular}{|l|l|l|l|}
\hline Variables & Loadings & \multicolumn{3}{l|}{$\begin{array}{l}\text { BCa bootstrap 95\% confidence } \\
\text { intervals }\end{array}$} \\
\hline RVP_A & $\mathbf{0 . 7 2 2}$ & 0.599 & 0.802 \\
\hline SWM Strategy & $\mathbf{0 . 5 7 4}$ & 0.407 & 0.674 \\
\hline PAL_TEA & $\mathbf{0 . 7 2 3}$ & 0.604 & 0.817 \\
\hline RVP_ML & $\mathbf{0 . 5 7 1}$ & 0.393 & 0.684 \\
\hline SWM_BE & $\mathbf{0 . 8 2 1}$ & 0.707 & 0.888 \\
\hline OTS_PSOFC & $\mathbf{0 . 8 3 0}$ & 0.743 & 0.887 \\
\hline IED_TEA & $\mathbf{0 . 5 7 9}$ & 0.438 & 0.700 \\
\hline ERT_MORL & $\mathbf{0 . 5 2 6}$ & 0.351 & 0.680 \\
\hline WLM & $\mathbf{0 . 7 0 3}$ & 0.578 & 0.816 \\
\hline VFT & $\mathbf{0 . 6 6 3}$ & 0.515 & 0.753 \\
\hline MMSE & $\mathbf{0 . 7 1 9}$ & 0.615 & 0.790 \\
\hline True Recall & $\mathbf{0 . 6 4 1}$ & 0.518 & 0.770 \\
\hline Kaiser-Meyer-Olkin test & 0.863 & 0.848 & 0.889 \\
\hline Unidimensional Congruence & 0.965 & 0.955 & 0.979 \\
\hline Explained Common Variance & 0.850 & 0.828 & 0.878 \\
\hline Mean item residual absolute loadings & 0.232 & 0.203 & 0.246 \\
\hline Goodness of Fit Index & 0.974 & 0.965 & 0.984 \\
\hline Generalized H index & 0.923 & 0.922 & 0.960 \\
\hline Weighted Root Mean Square Residual & 0.179 & 0.133 & 0.206 \\
\hline
\end{tabular}

PAL_TEA: Paired-association learning, total errors adjusted; RVP_A: Rapid visual information process test, A' prime; RVP_ML: Rapid visual information process test, median latency; SWM_BE: Spatial working memory, between errors; SWM_STR: Spatial working memory, strategy; OTS_PSOFC: One touch stockings of Cambridge, problems solved on first choice; IED_EDS: Intra/extradimensional set shifting, shift errors; IED_TEA: Intra/extradimensional set shifting, total errors adjusted; ERT_MORL: Emotional recognition test, median overall response latency; MMSE: Mini Mental State Examination; VFT: Verbal

Fluency

Test;

WLM:

World

List

memory. 


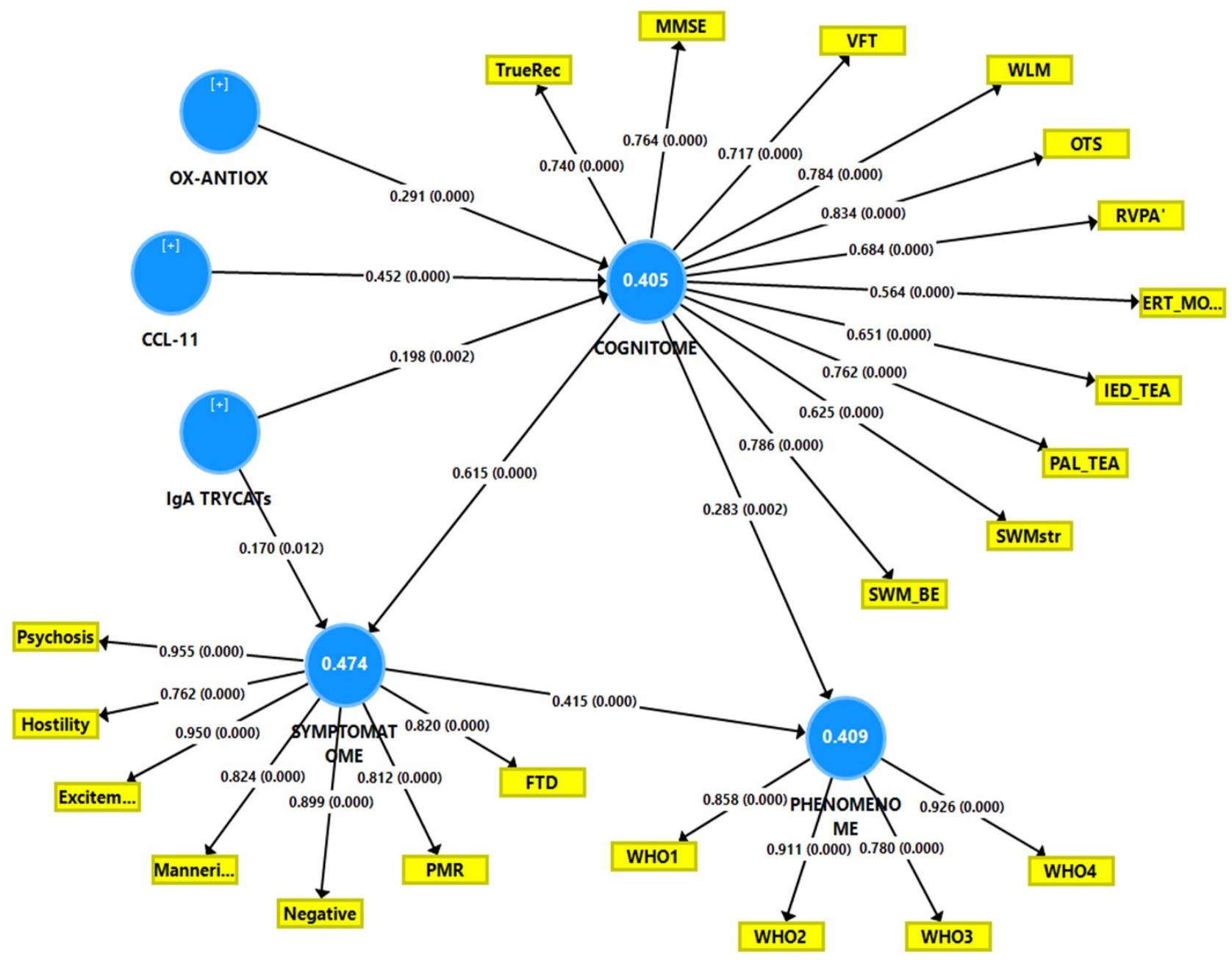

Figure 1. Results of Partial Least Squares analysis.

OX-ANTIOX: ratio between oxidative stress toxicity/antioxidant defenses; IgA TRYCATs: IgA directed against tryptophan catabolites.

TrueRec: True Recall; MMSE: Mini Mental State Examination; VFT: Verbal Fluency Test; WLM: World List memory.

OTS: One touch stockings of Cambridge, problems solved on first choice; RVPA': Rapid visual information process test, A' prime; ERT_MORL: Emotional recognition test, median overall response latency; IED_TEA: Intra/extradimensional set shifting, total errors adjusted; PAL_TEA: Paired-association learning, total errors adjusted; SWM_STR: Spatial working memory, strategy; SWM_BE: Spatial working memory, between errors.

Excitem: Excitement; Manneri: mannerism; Negative: negative symptoms; PMR: psychomotor retardation; FTD: formal thought disorders.

WHO1-4: World Health Organization Quality of Life Instrument-Abbreviated version domains: 1) physical health; 2) psychological health; 3) social relationships; and 4) environment. 


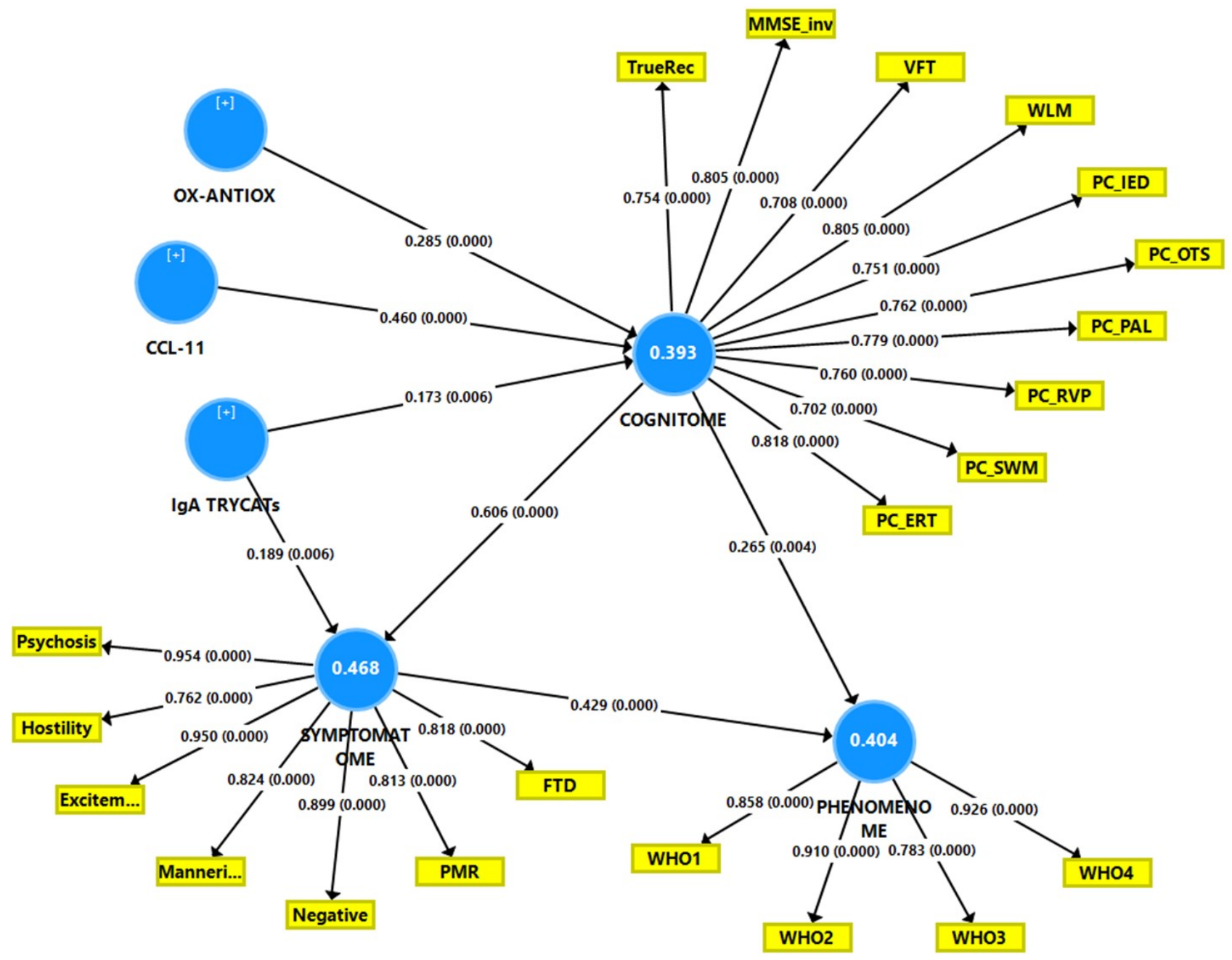

Figure 2. Results of Partial Least Squares analysis.

OX-ANTIOX: ratio between oxidative stress toxicity/antioxidant defenses; IgA TRYCATs: IgA directed against tryptophan catabolites.

TrueRec: True Recall; MMSE: Mini Mental State Examination; VFT: Verbal Fluency Test; WLM: World List memory.

PC_: first principal component extracted from various test scores; IED: Intra/extradimensional set shifting tests; OTS: One touch stockings of Cambridge tests; PAL: Paired-association learning; RVP: Rapid visual information process; SWM: Spatial working memory; ERT: Emotional recognition test. Excitem: Excitement; Manneri: mannerism; Negative: negative symptoms; PMR: psychomotor retardation; FTD: formal thought disorders.

WHO1-4: World Health Organization Quality of Life Instrument-Abbreviated version domains: 1) physical health; 2) psychological health; 3) social relationships; and 4) environment. 


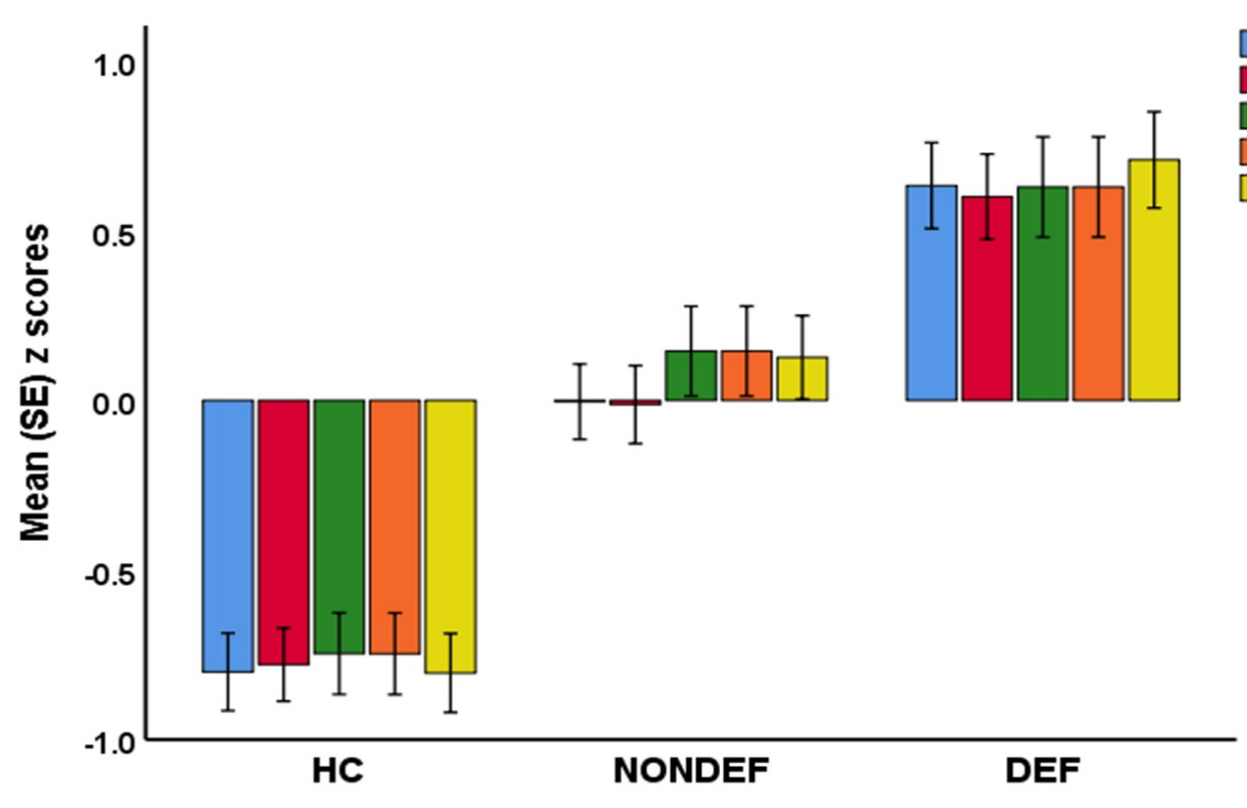

3CERAD

TCERAD

9CANTAB

$\square$ PC7CANTAB

$\square$ CERAD+CANTAB

Figure 3. Clustered bar graph with neurocognitive scores in healthy controls (HC), and schizophrenia patients with (DEF) and without (NONDEF) deficit.

3CERAD: $\mathrm{z}$ unit-weighted composite score of Word List memory + Verbal Fluency + True Recall scores; 4CERAD: composite score of 3CERAD + MMSE (Mini Mental State Examination); 9CANTAB: composite score of 9 key CANTAB tests; PC7CANTAB: Principal component extracted from various test of 7 different domains; CERAD+CANTAB: computed as z unit-weighted composite score of 3 CERAD + 9CANTAB. 


\section{ELECTRONIC SUPPLEMENTARY FILE 1 (ESF 1)}

ESF1, Table 1. Indices of the different symptom domains and biomarker composite scores used in the current study

\begin{tabular}{|c|c|}
\hline $\begin{array}{l}\text { Symptom domains } \\
\text { Biomarker scores }\end{array}$ & $\mathrm{Z}$ unit weighted composite scores \\
\hline Psychosis & $\begin{array}{l}\text { sum of z score of item } 1 \text { on the positive subscale of the PANSS (zPANNSP1, } \\
\text { delusion) plus zPANSSP3 (hallucinations) + zPANNSP6 (suspiciousness) plus } \\
\text { z score of item } 11 \text { of the BPRS (zBPRS11: suspiciousness) plus zBPRS12 } \\
\text { (hallucinatory behavior) plus zBPRS15 (unusual thought content). }\end{array}$ \\
\hline Hostility & $\begin{array}{l}\text { sum of zPANSSP7 (hostility) plus z-score of item } 14 \text { on the general } \\
\text { psychopathology scale of the PANSS (zPANSSG14: poor impulse control) } \\
\text { plus zBPRS10 (hostility) plus zBPRS14 (uncooperativeness). }\end{array}$ \\
\hline Excitement & $\begin{array}{l}\text { zPANNSP4 (excitement) plus zPANNSP5 } \\
\text { (grandiosity) plus zBPRS17 (excitement). }\end{array}$ \\
\hline Mannerism & zPANNSG5 plus zBPRS7 (both mannerism and posturing) \\
\hline $\begin{array}{l}\text { Formal thought } \\
\text { disorders }\end{array}$ & $\begin{array}{l}\text { zPANNSP2 (conceptual disorganization) plus item } 5 \text { of the PANNS negative } \\
\text { subscale (PANNSN5: difficulty in abstract thinking) plus zBPRS4 (item } 4 \text { of } \\
\text { the BPRS or conceptual disorganization) }\end{array}$ \\
\hline $\begin{array}{l}\text { Psychomotor } \\
\text { retardation }\end{array}$ & $\begin{array}{l}\text { z-score of HDRS item } 8 \text { (HDRS8: psychomotor retardation: slowness of } \\
\text { thought and speech, decreased motor activity, impaired inability to } \\
\text { concentrate) plus zPANSSG7 (reduction in motor activity as reflected in } \\
\text { slowing or lessening of movements and speech, diminished responsiveness to } \\
\text { stimuli and reduced body tone) plus zBPRS13 (reduction in energy level } \\
\text { evidenced in slowed movements). }\end{array}$ \\
\hline $\begin{array}{l}\text { NOX/PRO } \\
\text { TRYCAT }\end{array}$ & $\begin{array}{l}\text { Ratio of noxious TRYCATs / generally more protective (NOX/PRO) } \\
\text { TRYCATs computed as } z \text { score of IgA to picolinic acid }+z \text { IgA to xanthurenic } \\
\text { acid }+z \text { IgA 3-OH-kynurenine }-\mathrm{z} \text { IgA anthranilic acid }-\mathrm{z} \text { kynurenic acid. }\end{array}$ \\
\hline $\begin{array}{l}\text { OSTOX/ANTIOX } \\
\text { ratio }\end{array}$ & $\begin{array}{l}\text { Ratio of oxidative stress toxicity / antioxidants computed as: } z \text { ( } \mathrm{z} \text { lipid } \\
\text { hydroperoxides }+\mathrm{z} \text { malondialdehyde }+\mathrm{z} \text { advanced oxidation protein } \\
\text { products) }-\mathrm{z} \text { ( } \mathrm{z} \text { paraoxonase } 1 \text { activity }+\mathrm{z} \text { sulphydryl groups }+\mathrm{z} \text { total } \\
\text { radical trapping parameter (TRAP) }\end{array}$ \\
\hline
\end{tabular}

PANNS: Positive and Negative Syndrome Scale; BPRS: Brief Psychiatric Rating Scale (BPRS); HDRS: Hamilton Depression Rating Scale. 


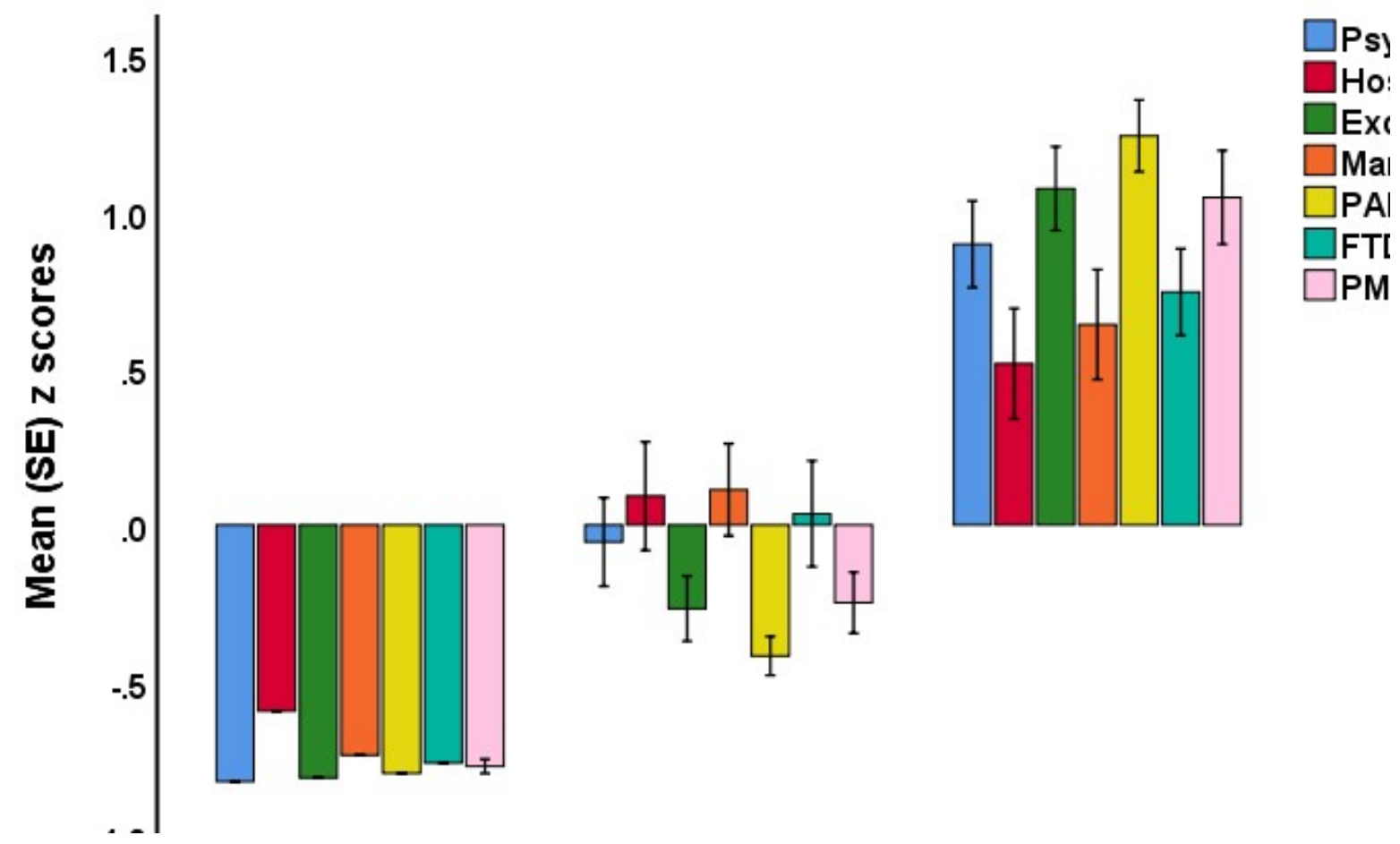

ESF 1, Figure 1. Clustered bar graph showing the symptomatome domains of schizophrenia

in healthy controls (HC) and patients with nondeficit (NONDEF) and deficit (DEF) schizophrenia.

PANNSneg: the negative subscale of the Positive and Negative Syndrome Scale; FTD: formal thought disorders; PMR: psychomotor retardation.

Multivariate GLM analysis adjusted for age, sex, and education shows a significant association between diagnosis and the symptom domains $(\mathrm{F}=22.13, \mathrm{df}=14 / 214, \mathrm{p}<0.001$, partial eta squared $=0.602$ ). Pairwise comparisons among treatment means showed that all domain scores were significantly different between the three classes (except hostility which did not differ between both schizophrenia groups). 

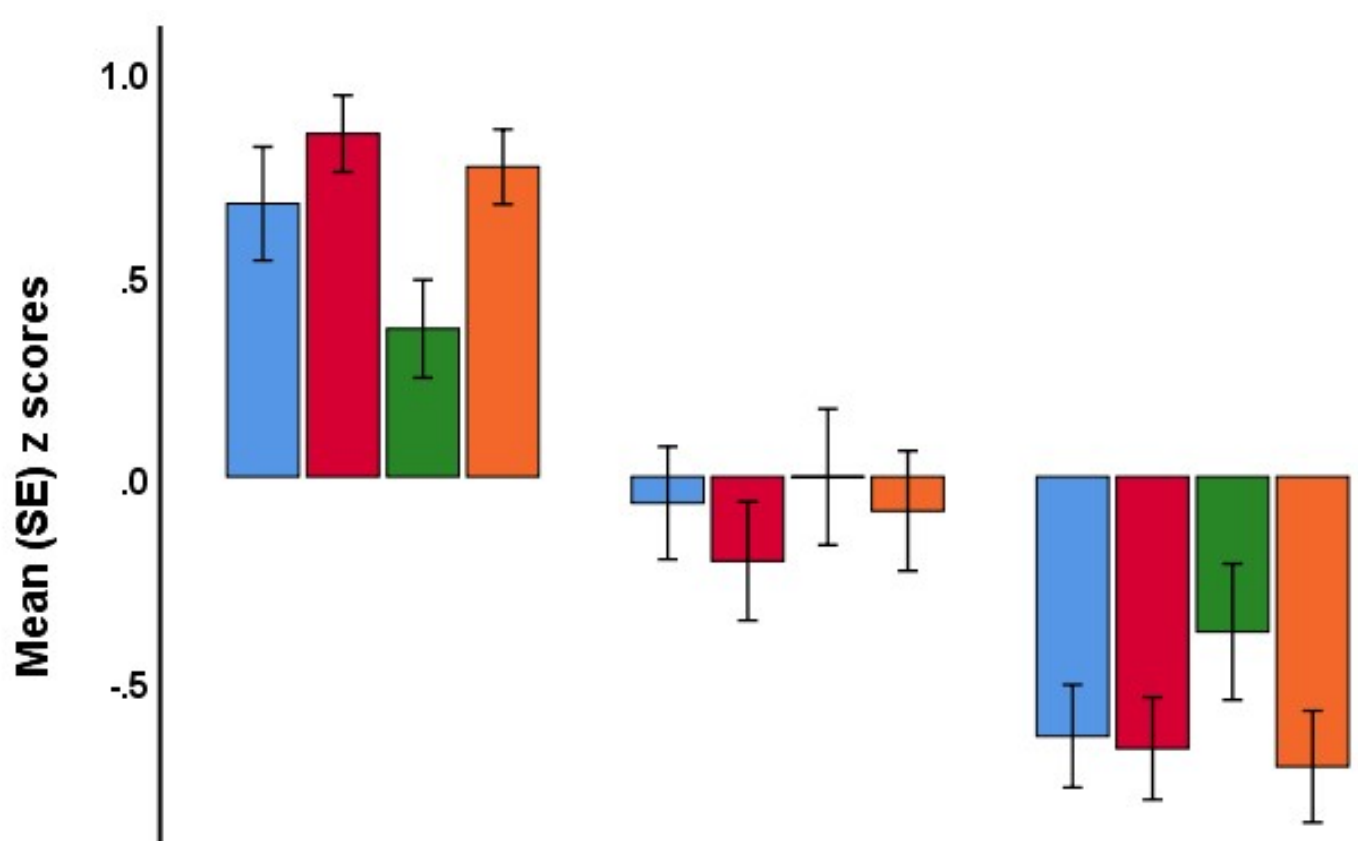

ESF 1, Figure 2. Clustered bar graph showing the World Health Organization Quality of Life scores (in $\mathrm{z}$ values) in healthy controls (HC) and patients with nondeficit (NONDEF) and deficit (DEF) schizophrenia.

Shown are the mean z scores of domain 1: physical health; domain 2: psychological health; domain 3: social relationships; and domain 4: environment.

Multivariate GLM analysis adjusted for age, sex, and education shows a significant association between diagnosis and the WHO-QoL domains $(\mathrm{F}=9.80, \mathrm{df}=8 / 218, \mathrm{p}<0.001$, partial eta squared $=0.265$ ). Pairwise comparisons among treatment means showed that all domain scores were significantly different between the three classes, except domain 3 which was different between HC and DEF only.

\section{ESF 1, Section 1: Effects of confounding variables.}

Univariate GLM analyses (with diagnosis, sex and education as additional explanatory variables) and false discovery rate $\mathrm{p}$ correction showed that age was significantly and positively associated with SWM_BE ( $\mathrm{p}=0.005)$, SWM_STR $(\mathrm{p}=0.007)$ and ERT_MORL $(\mathrm{p}=0.042)$ and significantly and negatively with OTS_PSOFC $(\mathrm{p}=0.005)$. Univariate GLM analyses (with diagnosis, sex, and age as additional explanatory variables) and false discovery rate $\mathrm{p}$ correction showed that years of education was significantly and positively associated with RVP_A $(p=0.002)$, OTS_PSOFC $(p=0.002)$ and negatively with PAL_TEA $(p=0.015), S W M \_B E-(p=0.015)$, and IED_TEA $(p=0.002)$. Tobacco use disorder $(F=1.86$, $\mathrm{df}=10 / 100, \mathrm{p}=0.060), \mathrm{BMI}(\mathrm{F}=1.01, \mathrm{df}=1 \overline{0} / 979, \mathrm{p}=0.442)$ and employment status $(\mathrm{F}=0.90$, $\mathrm{df}=10 / 100, \mathrm{p}=0.535)$ did not show a significant effect upon CANTAB performance, while the effects of diagnosis remained significant.

The effects of drug treatment of the participants were also investigated. Prescribed medications were risperidone: $n=32$, olanzapine: $n=5$, quetiapine $n=4$, clozapine: $n=10$, haloperidol $\mathrm{n}=10$, perphenazine $\mathrm{n}=19$, chlorpromazine $\mathrm{n}=4$, fluphenazine $\mathrm{n}=10$, antidepressants $n=28$, mood stabilizers $n=12$, anxiolytics $n=29$, and trihexyphenidyl $n=47$. Using univariate GLM analysis no significant effects of these drugs (or their dosages) could be found on any of the 9 CANTAB or 4 CERAD tests. 


\section{ELECTRONIC SUPPLEMENTARY FILE 2 (ESF 2)}

\section{ESF 2, Methods.}

\section{Table 1. All CANTAB tests performed in our study.}

a) Motor screening test (MOT). We analyzed 4 MOT variables, i.e. MOT mean latency (MOT-ML), MOT mean error (MOT-ME), MOT total correct (MOT-TC) and MOT total errors (MOT-TE).

b) Paired-association learning (PAL). We analyzed 11 PAL variables, i.e. PAL first trial memory score (PAL-FTMS), PAL mean errors to success (PAL-METS), PAL mean trials to success (PAL-MTTS), PAL number of patterns reached (PAL-NPR), PAL number of patterns succeeded (PAL-NPS), PAL stages completed (PAL-SC), PAL stages completed on first trial (PAL-SCFT), PAL total errors (PAL-TE), PAL total errors adjusted (PAL-TEA), PAL total trials (PAL-TT) and PAL total trials adjusted (PAL-TTA).

c) One touch stockings of Cambridge (OTS). We analyzed 6 OTS outcome measures, i.e. OTS mean latency to correct (OTS-MLTC), OTS mean latency to first choice (OTSMLTFC), OTS mean choices to correct (OTS-MCTC), OTS probability of error given correct (OTS-PEGC), OTS probability of error given error (OTS-PEGE) and OTS probability solved on first choice (OTS-PSOFC).

d) Rapid visual information process test (RVP). We used 11 RVP outcome measures, i.e. RVP A, RVP probability of false alarm (RVP-PFA), RVP probability of hit (RVP-PH), RVP probability of hit blocks 1-7 (RVP-PHB1-7), RVP mean latency (RVP-ML), RVP total correct rejections (RVP-TCR), RVP total false alarms (RVP-TFA), RVP total hits (RVP-TH), RVP total hits blocks 1-7 (RVP-THB1-7), RVP total misses (RVP-TM), RVP total misses blocks 1-7 (RVP-TMB1-7).

e) Spatial working memory (SWM). We analyzed 9 SWM outcome measures, i.e. SWM between errors (SWM-BE), SWM between errors 4 boxes (SWM-BE4B), SWM strategy (SWM-STR), SWM strategy 4-10 boxes (SWM-STR4-10B), SWM mean time to first response (SWM-MTFR), SWM mean time to last response (SWM-MTLR), SWM mean token preparation time (SWM-MTPT), SWM total errors (SWM-TE) and SWM total error 4 boxes (SWM-TE4B).

f) Intra/extradimensional set shifting (IED). We assessed 10 outcome measures, i.e. IED completed stage errors (IED-CSE), IED complete stage trials (EID-CST), IED errors block 1 (IED-EB1), IED Pre-ED errors (IED-PEE), IED stages completed (IED-SC), IED total errors (IED-TE), IED total errors adjusted (IED-TEA), IED total latency (IED-TL), IED total trials (IED-TT) and IED total trials adjusted (IED-TTA).

g) Emotional recognition test (ERT). We analyzed 3 ERT outcome measures, i.e. ERT mean overall response latency (ERT-MORL). ERT percent correct (ERT-PC), and ERT total number correct (ERT-TNC).

\section{ESF 2, Statistics}

Extraction of CANTAB PC subdomains.

We used factor analyses, principal component (PC) method (using SPSS25) to reduce the number of CANTAB variables, namely 4 MOT, 11 PAL, 11 RVP, 9 SWM, 6 OTS, 10 IED, and 3 ERT tests into 7 interpretable PCs reflecting the same subdomains. Towards this end we have extracted the first PC of each CANTAB subtest. The first PC of the 4 MOT tests explained $65.6 \%$ of the variance, the first PC in the PAL data explained $76.6 \%$ of the variance, RVP: $76.8 \%$, SWM: $64.5 \%$, OTS: 68.5\%, IED: $48.7 \%$ and ERT: $75.3 \%$. As such 
we have delineated seven interpretable factors, which reflect the total variability in the CANTAB subdomains. Subsequently, we have entered the seven first PC scores in multivariate GLM analysis. If the overall GLM analysis performed on the 7 PCs showed significant effects of diagnosis and the tests of between-subject effects on a particular PC were significant, we also examined the effects of diagnosis on all CANTAB tests comprising that subdomain. We also examined whether a latent trait could be extracted from these 7 PCs and found that a first PC explained $57.2 \%$ of the variance in the 7 CANTAB tests which were all highly loaded on this first PC (all $>0.750$, except PC MOT which showed a loading of $0.539)$.

\section{ESF 2, Results}

Table 1 shows a significant association between diagnosis and the PAL performance $(\mathrm{F}=2.00, \mathrm{df}=22 / 208, \mathrm{p}=0.007)$ after controlling for age $(\mathrm{F}=2.06, \mathrm{df}=11 / 103, \mathrm{p}=0.030)$, sex $(\mathrm{F}=1.91, \mathrm{df}=11 / 103, \mathrm{p}=0.047)$ and education $(\mathrm{F}=2.07, \mathrm{df}=11 / 103, \mathrm{p}=0.029)$. Table 3 shows the estimated marginal mean (SE) values of the 11 PAL measurements and the tests of between-subject effects for each of the measurements. Tests for between-subject effects showed significant effects of diagnosis on all 11 PAL variables. PAL-FTMS and PAL-SCFT scores were significantly different between the three groups with increasing impairments from controls to nondeficit to deficit schizophrenia. The performance assessed by PAL-NPR, PAL-NPS, PAL-TEA, and PAL-TTA were significantly worse in deficit schizophrenia than in controls and nondeficit schizophrenia. PAL-MTTS, PAL-SC and PAL-TT scores were significantly poorer in deficit schizophrenia versus controls, while results in nondeficit schizophrenia occupied an intermediate position. PAL-METS and PAL-TE were significantly more affected in schizophrenia than in controls.

ESF 2, Results, Table 1. Results of multivariate GLM analyses which examines the associations between the principal components (PCs) extracted from 7 CANTAB domains and diagnosis.

\begin{tabular}{llllllc}
\hline Test type & Dependent & Explanatory & F & Df & P & Partial Eta \\
& Variables & variables & & & & Squared \\
\hline Multivariate & All 7 PCs & Diagnosis & 3.34 & $14 / 206$ & $<0.001$ & 0.175 \\
& & Gender & 1.24 & $7 / 103$ & 0.287 & 0.078 \\
& & Education & 7.16 & $7 / 103$ & $<0.001$ & 0.327 \\
& & Age & 5.19 & $7 / 103$ & $<0.001$ & 0.261 \\
\hline $\begin{array}{l}\text { Tests for } \\
\text { between- }\end{array}$ & PC MOT & Diagnosis & 1.51 & $2 / 109$ & 0.226 & 0.027 \\
subject effects & PC PAL & Diagnosis & 8.71 & $2 / 109$ & $<0.001$ & 0.138 \\
& & & & & & \\
& PC RVI & Diagnosis & 6.94 & $2 / 109$ & 0.001 & 0.113 \\
& PC SWM & Diagnosis & 17.34 & $2 / 109$ & $<0.001$ & 0.241 \\
& PC OTS & Diagnosis & 14.43 & $2 / 109$ & $<0.001$ & 0.209
\end{tabular}




$\begin{array}{llcccc}\text { PC IED } & \text { Diagnosis } & 5.08 & 2 / 109 & 0.008 & 0.085 \\ \text { PC ERT } & \text { Diagnosis } & 11.50 & 2 / 109 & <0.001 & 0.174\end{array}$

Diagnosis: 3 groups, namely healthy controls and schizophrenia with and without the deficit syndrome.

PC MOT: Motor screening test to screen visual, movement and comprehension; PC PAL: Paired-association learning to assess visual memory, episodic memory and learning; PC RVP: Rapid visual information process test to assess visual sustained attention; PC SWM: Spatial working memory to assess working memory and strategy use; PC OTS: One touch stockings of Cambridge to assess spatial planning; PC IED: Intra/extradimensional set shifting to assess rule acquisition and attention set shifting; PC ERT: Emotional recognition test to interpret facial expression of emotion.

ESF 2, Results, Table 2. Model-generated estimated marginal means (SE) of the principal components (PCs) extracted from 7 CANTAB domains, in normal controls and schizophrenia patient with and without deficit schizophrenia (SCZ)

\begin{tabular}{|l|c|c|c|}
\hline \multicolumn{1}{|c|}{ CANTAB } & Normal Control $^{\mathbf{A}}$ & Nondeficit SCZ $^{\mathbf{B}}$ & Deficit SCZ $^{\mathbf{C}}$ \\
\hline PC MOT & $-0.21(0.13)$ & $-0.13(0.12)$ & $0.10(0.13)$ \\
\hline PC PAL & $-0.46(0.19)^{\mathrm{B}, \mathrm{C}}$ & $-0.05(0.13)^{\mathrm{A}, \mathrm{C}}$ & $0.38(0.19)^{\mathrm{A}, \mathrm{B}}$ \\
\hline PC RVP & $0.38(0.13)^{\mathrm{C}}$ & $0.06(0.13)^{\mathrm{C}}$ & $-0.33(0.13)^{\mathrm{A}, \mathrm{B}}$ \\
\hline PC SWM & $-0.62(0.13)^{\mathrm{B}, \mathrm{C}}$ & $0.09(0.12)^{\mathrm{A}, \mathrm{C}}$ & $0.47(0.13)^{\mathrm{A}, \mathrm{B}}$ \\
\hline PC OTS & $-0.57(0.13)^{\mathrm{B}, \mathrm{C}}$ & $0.10(0.12)^{\mathrm{A}}$ & $0.38(0.13)^{\mathrm{A}}$ \\
\hline PC IED & $-0.41(0.14)^{\mathrm{B}, \mathrm{C}}$ & $0.12(0.13)^{\mathrm{A}}$ & $0.15(0.14)^{\mathrm{A}}$ \\
\hline PC ERT & $0.47(0.12)^{\mathrm{B}, \mathrm{C}}$ & $-0.03(0.12)^{\mathrm{A}, \mathrm{C}}$ & $-0.37(0.12)^{\mathrm{A}, \mathrm{B}}$ \\
\hline
\end{tabular}

All results of GLM analysis after covarying for age, sex, and education. A,B,C: pairwise comparisons among treatment means

PC: first principal component extracted from various CANTAB domain tests; PC MOT: Motor screening test to screen visual, movement and comprehension; PC PAL: Pairedassociation learning to assess visual memory, episodic memory and learning; PC RVP: Rapid visual information process test to assess visual sustained attention; PC SWM: Spatial working memory to assess working memory and strategy use; PC OTS: One touch stockings of Cambridge to assess spatial planning; PC IED: Intra/extradimensional set shifting to assess rule acquisition and attention set shifting; PC ERT: Emotional recognition test to interpret facial expression of emotion. 
Table 3 shows a significant association between diagnosis and PAL subtests $(\mathrm{F}=2.00$, $\mathrm{df}=22 / 208, \mathrm{p}=0.007)$ after controlling for age $(\mathrm{F}=2.06, \mathrm{df}=11 / 103, \mathrm{p}=0.030)$, sex $(\mathrm{F}=1.91$, $\mathrm{df}=11 / 103, \mathrm{p}=0.047)$ and education $(\mathrm{F}=2.07, \mathrm{df}=11 / 103, \mathrm{p}=0.029)$. Table 3 shows the estimated marginal mean (SE) values of the 11 PAL measurements and the tests of betweensubject effects for each of the measurements. Tests for between-subject effects showed significant effects of diagnosis on all 11 PAL variables (PAL_TEA is shown in Table 2-3 of the main text). PAL-FTMS and PAL-SCFT scores were significantly different between the three groups with increasing impairments from controls to nondeficit to deficit schizophrenia. The cognitive performance as assessed by PAL-NPR, PAL-NPS, and PAL-TTA was significantly worse in deficit schizophrenia than in controls and nondeficit schizophrenia. PAL-MTTS, PAL-SC and PAL-TT scores were significantly poorer in deficit schizophrenia versus controls, while patients with nondeficit schizophrenia occupied an intermediate position. PAL-METS and PAL-TE were significantly more affected in schizophrenia than in controls.

ESF 2, Results, Table 3. Results of multivariate GLM analysis examining the associations between 11 paired-association learning (PAL) test scores and diagnosis [entered as 3 groups: normal controls versus nondeficit schizophrenia (SCZ) versus deficit schizophrenia] while controlling for age, gender and education.

\begin{tabular}{|l|l|l|l|l|l|l|}
\hline Measurements & Controls $^{\mathbf{A}}$ & $\begin{array}{l}\text { Nondeficit } \\
\mathbf{S C Z}^{\mathbf{B}}\end{array}$ & $\begin{array}{l}\text { Deficit } \\
\text { SCZ }^{\mathbf{C}}\end{array}$ & $\mathbf{F}$ & df & P \\
\hline PAL-FTMS & $17.3(0.8)^{\mathrm{B}, \mathrm{C}}$ & $14.6(0.8)^{\mathrm{A}, \mathrm{C}}$ & $\begin{array}{l}11.2(0.8) \\
\mathrm{A}, \mathrm{B}\end{array}$ & 14.00 & $2 / 113$ & $<0.001$ \\
\hline PAL-METS & $2.8(0.5)^{\mathrm{B}, \mathrm{C}}$ & $4.3(0.5)^{\mathrm{A}}$ & $5.5(0.5)^{\mathrm{A}}$ & 6.28 & $2 / 113$ & 0.003 \\
\hline PAL-MTTS & $2.1(0.2)^{\mathrm{C}}$ & $2.5(0.2)$ & $3.1(0.2)^{\mathrm{A}}$ & 4.90 & $3 / 113$ & 0.009 \\
\hline PAL-NPR & $7.5(0.2)^{\mathrm{C}}$ & $7.4(0.2)^{\mathrm{C}}$ & $\begin{array}{l}6.6(0.2) \\
\mathrm{A}, \mathrm{B}\end{array}$ & 4.15 & $2 / 113$ & 0.018 \\
\hline PAL-NPS & $7.1(0.4)^{\mathrm{C}}$ & $6.8(0.3)^{\mathrm{C}}$ & $\begin{array}{l}5.8(0.3) \\
\mathrm{A}, \mathrm{B}\end{array}$ & 3.96 & $2 / 113$ & 0.022 \\
\hline PAL-SC & $7.5(0.2)^{\mathrm{C}}$ & $7.3(0.2)$ & $6.7(0.2)^{\mathrm{A}}$ & 3.52 & $2 / 113$ & 0.033 \\
\hline PAL-SCFT & $5.5(0.2)^{\mathrm{B}, \mathrm{C}}$ & $4.7(0.2)^{\mathrm{A}, \mathrm{C}}$ & $\begin{array}{l}3.7(0.2) \\
\mathrm{A}, \mathrm{B}\end{array}$ & 13.16 & $2 / 113$ & 0.001 \\
\hline PAL-TE & $18.3(3.3)^{\mathrm{B}, \mathrm{C}}$ & $28.1(3.1)^{\mathrm{A}}$ & $33.4(3.2)^{\mathrm{A}}$ & 5.41 & $2 / 113$ & 0.006 \\
\hline PAL-TT & $14.1(0.9)^{\mathrm{C}}$ & $16.4(0.8)$ & $18.3(0.8)^{\mathrm{A}}$ & 6.28 & $2 / 113$ & 0.003 \\
\hline PAL-TTA & $17.0(1.9)^{\mathrm{C}}$ & $19.8(1.8)^{\mathrm{C}}$ & $\begin{array}{l}26.7(1.8) \\
\mathrm{A}, \mathrm{B}\end{array}$ & 7.18 & $2 / 113$ & 0.001 \\
\hline
\end{tabular}

All results are shown as estimated marginal means (SE) obtained after multivariate GLM analysis. F values: results of tests for between-subject effects performed when the multivariate analysis was significant. 
$\mathrm{A}, \mathrm{B}, \mathrm{C}$ : pairwise comparisons among treatment means. ${ }^{\mathrm{A}}$ : significantly different from controls, B: from nondeficit schizophrenia, C: from deficit schizophrenia (protected post-hoc tests)

11 PAL variables are measured, i.e. PAL first trial memory score (FTMS), PAL mean errors to success (METS), PAL mean trials to success (MTTS), PAL number of patterns reached (NPR), PAL number of patterns succeeded (NPS), PAL stages completed (SC), PAL stages completed on first trial (SCFT), PAL total errors (TE), PAL total errors adjusted (TEA), PAL total trials (TT) and PAL total trial adjusted (TTA)

Table 4 shows that there was a significant association between diagnosis and the 6 OTS $(\mathrm{F}=4.49, \mathrm{df}=12 / 216, \mathrm{p}<0.001)$ scores after controlling for the effects of gender, education, and age (results of multivariate GLM). OTS-MLTC was significantly lower in deficit schizophrenia than in controls, while nondeficit patients took up an intermediate position. OTS-MCTC, OTC-PEGC, and OTS-PEGE are higher in both schizophrenia subgroups as compared with controls.

Table 4 shows that there was a significant association between the diagnosis and RVP values $(\mathrm{F}=2.70, \mathrm{df}=14 / 210, \mathrm{p}=0.001$; results of multivariate $\mathrm{GLM})$. Tests for between-subject effects showed significant associations between the diagnosis and all RVP measurements (RVP-A and RVP_ML are shown in Tables 2-3 of the main text). RVP-PFA, RVP-PH, RVPTCR, RVP-TFA, RVP-TH, and RVP-TM are significantly more disordered in deficit schizophrenia as compared with nondeficit schizophrenia and controls. RVP-PHB1-7, RVPTHB1-7, and RVP-TMB1-7 were significantly more affected in deficit schizophrenia than in controls, while patients with nondeficit schizophrenia occupied an intermediate position.

ESF 2, Results, Table 4. Results of multivariate GLM analyses with the 6 One touch of stocking of Cambridge (OTS) scores examining the associations between 11 Rapid Visual Information Processing (RVP) and diagnosis [entered as 3 groups: normal controls versus nondeficit schizophrenia (SCZ) versus deficit schizophrenia] while controlling for age, gender and education.

\begin{tabular}{|l|l|l|l|l|l|l|}
\hline Measurements & Controls $^{\mathbf{A}}$ & $\begin{array}{l}\text { Nondeficit } \\
\mathbf{S C Z}^{\mathbf{B}}\end{array}$ & $\begin{array}{l}\text { Deficit } \\
\mathbf{S C Z}^{\mathbf{C}}\end{array}$ & $\mathbf{F}$ & df & P \\
\hline OTS-MLTC & $\begin{array}{l}25439(1939) \\
\mathrm{C}\end{array}$ & $20945(1838)$ & $\begin{array}{l}18469 \\
(1869)^{\mathrm{A}}\end{array}$ & 3.33 & $2 / 113$ & 0.040 \\
\hline OTS-MLTFC & $16849(1555)$ & $14365(1474)$ & $\begin{array}{l}12644 \\
(1498)\end{array}$ & 1.86 & $2 / 113$ & 0.161 \\
\hline OTS-MCTC & $2.1(0.1)^{\mathrm{B}, \mathrm{C}}$ & $2.6(1.1)^{\mathrm{A}}$ & $2.8(1.1)^{\mathrm{A}}$ & 7.65 & $2 / 113$ & 0.001 \\
\hline OTS-PEGC & $\begin{array}{l}0.42(0.05) \\
\mathrm{B}, \mathrm{C}\end{array}$ & $0.69(0.04)^{\mathrm{A}}$ & $\begin{array}{l}0.70(0.04) \\
\mathrm{A}\end{array}$ & 12.72 & $2 / 113$ & $<0.001$ \\
\hline OTS-PEGE & $\begin{array}{l}0.36(0.04) \\
\mathrm{B}, \mathrm{C}\end{array}$ & $0.59(0.04)^{\mathrm{A}}$ & $\begin{array}{l}0.64(0.04) \\
\mathrm{A}\end{array}$ & 15.04 & $2 / 113$ & $<0.001$ \\
\hline RVP-PFA & $\begin{array}{l}0.008(0.007) \\
\mathrm{C}\end{array}$ & $\begin{array}{l}0.013(0.006) \\
\mathrm{C}\end{array}$ & $\begin{array}{l}0.032 \\
(0.006)^{\mathrm{A}, \mathrm{B}}\end{array}$ & 3.71 & $2 / 111$ & 0.027 \\
\hline
\end{tabular}




\begin{tabular}{|l|l|l|l|l|l|l|}
\hline RVP-PH & $0.88(0.03)^{\mathrm{C}}$ & $0.82(0.03)^{\mathrm{C}}$ & $\begin{array}{l}0.72(0.02) \\
\mathrm{A}, \mathrm{B}\end{array}$ & 7.02 & $2 / 111$ & 0.001 \\
\hline RVP-PHB1-7 & $0.88(0.02)^{\mathrm{C}}$ & $0.83(0.02)$ & $\begin{array}{l}0.77(0.02) \\
\mathrm{A}\end{array}$ & 5.00 & $2 / 111$ & 0.008 \\
\hline RVP-TCR & $526.7(5.6)^{\mathrm{C}}$ & $515.7(5.2)^{\mathrm{C}}$ & $\begin{array}{l}493.0(5.4) \\
\mathrm{A}, \mathrm{B}\end{array}$ & 9.89 & $2 / 111$ & $<0.001$ \\
\hline RVP-TFA & $4.0(3.3)^{\mathrm{C}}$ & $6.5(3.0)^{\mathrm{C}}$ & $\begin{array}{l}15.7(3.1) \\
\mathrm{A}, \mathrm{B}\end{array}$ & 3.80 & $2 / 111$ & 0.025 \\
\hline RVP-TH & $47.4(1.7)^{\mathrm{C}}$ & $44.3(1.6)^{\mathrm{C}}$ & $\begin{array}{l}38.6(1.6) \\
\mathrm{A}, \mathrm{B}\end{array}$ & 7.02 & $2 / 111$ & 0.001 \\
\hline RVP-THB1-7 & $47.4(1.3)^{\mathrm{C}}$ & $44.8(1.2)$ & $41.6(1.3)^{\mathrm{A}}$ & 4.50 & $2 / 111$ & 0.008 \\
\hline RVP-TM & $6.6(1.7)^{\mathrm{C}}$ & $9.7(1.6)^{\mathrm{C}}$ & $\begin{array}{l}15.3(1.6) \\
\mathrm{A}, \mathrm{B}\end{array}$ & 7.02 & $2 / 111$ & 0.001 \\
\hline RVP-TMB1-7 & $6.6(1.3)^{\mathrm{C}}$ & $9.2(1.2)$ & $12.4(1.3)^{\mathrm{A}}$ & 4.50 & $2 / 111$ & 0.008 \\
\hline
\end{tabular}

All results are shown as estimated marginal means obtained after multivariate GLM analysis. F values: results of tests for between-subject effects performed when the multivariate analysis is significant. ${ }^{\mathrm{A}, \mathrm{B}, \mathrm{C}}$ : pairwise comparisons among treatment means. ${ }^{\mathrm{A}}$ : significantly different from controls, ${ }^{\text {B: }}$ from nondeficit schizophrenia, ${ }^{\text {C: }}$ from deficit schizophrenia (protected posthoc tests)

One Touch Stockings of Cambridge (OTS); OTS mean latency to correct (MLTC), OTS mean latency to first choice (MLTFC), OTS mean choices to correct (MCTC), OTS probability of error given correct (PEGC), and OTS probability of error given error (PEGE).

Rapid visual information process test (RVP) variables, RVP probability of false alarm (PFA), RVP probability of hit (PH), RVP probability of hit blocks 1-7 (PHB1-7), RVP total correct rejections (TCR), RVP total false alarms (TFA), RVP total hits (TH), RVP total hits blocks 17 (THB1-7), RVP total misses (TM), RVP total misses blocks 1-7 (TMB1-7).

Table 5 shows that there was a significant association between the diagnosis and 9 SWM measurements $(\mathrm{F}=2.97, \mathrm{df}=18 / 210, \mathrm{p}<0.001$, results of multivariate $\mathrm{GLM})$ after controlling for age, sex and education (results on SWM_BE and SWM_STR are shown in Tables 2-3 of the main text). Tests for between-subject effects showed that SWM-BE4B, and SWM-STR4$10 \mathrm{~B}$ were significantly more negatively affected in both schizophrenia groups than in controls. SWM-MTFR, SWM-MTLR, SWM-MTPT and SWM-TE4B showed a significantly worse performance of deficit schizophrenia patients than of controls. SWM-TE was significantly different between the three study groups and increased from controls to nondeficit schizophrenia to deficit schizophrenia.

Table 5 shows that there was a significant association between the diagnosis and the 10 IED measurements $(\mathrm{F}=2.45, \mathrm{df}=16 / 210, \mathrm{p}=0.002$, results of multivariate GLM analysis; results on IED_TEA and IED_EDS are shown in tables 2-3 of the main text). IED-CSE, IED-TE, and IED-TT were significantly higher, showing worse performance, in both schizophrenic groups than in controls. IED total latency -TL was significantly greater in deficit schizophrenia as compared with controls and nondeficit schizophrenia. IED-TTA was significantly greater 
(worse result) in deficit schizophrenia than in controls, whereas patients with nondeficit schizophrenia took up an intermediate position.

Table 5 shows that there was a highly significant association between the diagnosis and 3 ERT measurements $(\mathrm{F}=6.84, \mathrm{df}=4 / 226, \mathrm{p}<0.001)$. Table $2-3$ in the main text showed that ERT median overall response latency (MORL) was significantly different between the three groups and increased from controls to nondeficit to deficit schizophrenia. ERT-PC and ERTTNC (correct results) were significantly lower in both patient groups than in controls.

ESF 2, Results, Table 5. Results of multivariate GLM analysis with the 10 Spatial Working Memory (SWM), 10 Intra-Extra Dimensional Set Shift (IED) and 3 Emotion Recognition Task (ERT) CANTAB measurements as dependent variables and diagnosis as primary explanatory variable [entered as 3 groups: normal controls versus nondeficit schizophrenia (SCZ) versus deficit schizophrenia] while controlling for age, gender and education.

\begin{tabular}{|c|c|c|c|c|c|c|}
\hline Measurements & Controls $^{\mathrm{A}}$ & $\begin{array}{l}\text { Nondeficit } \\
\text { SCZ }^{\text {B }}\end{array}$ & $\begin{array}{l}\text { Deficit SCZ } \\
\text { C }\end{array}$ & $\mathbf{F}$ & df & $\mathbf{p}$ \\
\hline SWM-BE4B & $2.2(0.6)^{\mathrm{B}, \mathrm{C}}$ & $4.2(0.6)^{\mathrm{A}}$ & $5.4(0.6)^{A}$ & 5.81 & $2 / 113$ & 0.004 \\
\hline $\begin{array}{l}\text { SWM-STR4- } \\
\text { 10B }\end{array}$ & $44.0(1.0)^{\mathrm{B}, \mathrm{C}}$ & $49.3(0.9)^{\mathrm{A}}$ & $51.1(0.9)^{\mathrm{A}}$ & 14.96 & $2 / 113$ & $<0.001$ \\
\hline SWM-MTFR & $2420(302)^{\mathrm{C}}$ & $2756(286)$ & $3348(291)^{\mathrm{A}}$ & 2.49 & $2 / 113$ & 0.088 \\
\hline SWM-MTLR & $\begin{array}{l}31521 \\
(1977)^{\mathrm{C}}\end{array}$ & $34740(1871)$ & $\begin{array}{l}39775 \\
(1905)^{\mathrm{A}}\end{array}$ & 4.53 & $2 / 113$ & 0.013 \\
\hline SWM-MTPT & $1444(126)^{\mathrm{C}}$ & 1573 (119) & $1848(122)^{\mathrm{A}}$ & 2.76 & $2 / 113$ & 0.068 \\
\hline SWM-TE & $32.7(3.3)^{\mathrm{B}, \mathrm{C}}$ & $51.6(3.1)^{\mathrm{A}, \mathrm{C}}$ & $60.4(3.2)^{\mathrm{B}, \mathrm{C}}$ & 18.65 & $2 / 113$ & $<0.001$ \\
\hline SWM-TE4B & $2.7(0.8)^{\mathrm{C}}$ & $4.2(0.7)$ & $6.0(0.7)^{\mathrm{A}}$ & 4.74 & $2 / 113$ & 0.011 \\
\hline SWM-TE4-10B & $32.7(3.3)^{\mathrm{B}, \mathrm{C}}$ & $51.6(3.1)^{\mathrm{A}, \mathrm{C}}$ & $60.4(3.2)^{\mathrm{B}, \mathrm{C}}$ & 18.65 & $2 / 113$ & $<0.001$ \\
\hline IED-CSE & $8.8(1.7)^{\mathrm{B}, \mathrm{C}}$ & $16.0(1.7)^{\mathrm{A}}$ & $14.1(1.7)^{\mathrm{A}}$ & 4.47 & $2 / 112$ & 0.014 \\
\hline IED-CST & $60.0(4.2)$ & $71.3(4.0)$ & $66.9(4.1)$ & 3.05 & $2 / 112$ & 0.052 \\
\hline IED-EB1 & $1.14(0.66)$ & $2.53(0.63)$ & $2.60(0.64)$ & 1.55 & $2 / 112$ & 0.218 \\
\hline IED-PEE & $8.5(2.3)$ & $15.7(2.2)$ & $13.4(2.2)$ & 2.61 & $2 / 112$ & 0.078 \\
\hline IED-SC & $7.4(0.3)$ & $7.4(0.3)$ & $6.9(0.3)$ & 1.02 & $2 / 112$ & 0.365 \\
\hline IED-TE & $25.2(2.1)^{\mathrm{B}, \mathrm{C}}$ & $35.9(2.0)^{\mathrm{A}}$ & $36.2(2.1)^{\mathrm{A}}$ & 8.44 & $2 / 112$ & $<0.001$ \\
\hline IED-TL & $\begin{array}{l}133042 \\
(18701)^{\mathrm{C}}\end{array}$ & $\begin{array}{l}172280 \\
(17718)^{\mathrm{C}}\end{array}$ & $\begin{array}{l}228994 \\
(18232)^{\mathrm{A}, \mathrm{B}}\end{array}$ & 6.76 & $2 / 112$ & 0.002 \\
\hline IED-TT & $85.2(4.0)^{\mathrm{B}, \mathrm{C}}$ & $104.7(3.7)^{\mathrm{A}}$ & $108.8(3.9)^{\mathrm{A}}$ & 10.14 & $2 / 112$ & $<0.001$ \\
\hline
\end{tabular}


30

\begin{tabular}{|l|l|l|l|l|l|l|}
\hline IED-TTA & $\begin{array}{l}135.9(11.7) \\
\mathrm{C}\end{array}$ & $153.8(11.1)$ & $\begin{array}{l}170.6(11.4) \\
\mathrm{A}\end{array}$ & 2.21 & $2 / 112$ & 0.115 \\
\hline & & & & & & \\
\hline ERT-PC & $48.3(1.8)^{\mathrm{B}, \mathrm{C}}$ & $41.6(1.7)^{\mathrm{A}}$ & $37.8(1.8)^{\mathrm{A}}$ & 8.34 & $2 / 114$ & $<0.001$ \\
\hline ERT-TNC & $86.9(3.3)^{\mathrm{B}, \mathrm{C}}$ & $74.9(3.3)^{\mathrm{A}}$ & $68.1(3.2)^{\mathrm{A}}$ & 8.34 & $2 / 114$ & $<0.001$ \\
\hline
\end{tabular}

All results are shown as estimated marginal means obtained after multivariate GLM analysis. F values: results of tests for between-subject effects performed when the multivariate analysis is significant ${ }^{\mathrm{A}, \mathrm{B}, \mathrm{C}}$ : pairwise comparisons among treatment means. ${ }^{\mathrm{A}}$ : significantly different from controls, ${ }^{B}$ from nondeficit schizophrenia, ${ }^{C}$ from deficit schizophrenia (protected posthoc tests)

SWM between errors 4 boxes (BE4B), SWM between errors 4-10 boxes (BE4-10B), SWM strategy 4-10 boxes (STR4-10B), SWM mean time to first response (MTFR), SWM mean time to last response (MTLR), SWM mean token time preparation time (MTTPT), SWM total errors (TE), SWM total error 4 boxes (TE4B) and SWM total errors 4-10 boxes (TE410B).

IED completed stage errors (CSE), IED complete stage trials (CST), IED errors block 1 (EB1), IED Pre-ED errors (PEE), IED stages completed (SC), IED total errors (TE), IED total latency (TL), IED total trials (TT) and IED total trials adjusted (TTA).

ERT total trials adjusted (TTA), ERT total number correct (TNC).

Table 6 shows the results of two different automatic stepwise logistic regression analyses with diagnosis as dependent variable and the CANTAB measurements as explanatory variables. As explanatory variables, we selected the three most significant PAL, OTS, RVP, SWM and IED scores and ERT-MORL. Regression \# 1a shows that gender and three CANTAB variables were significantly associated with schizophrenia, namely SWM-BE, IED-TT and ERT-MORL $\left(\mathrm{X}^{2}=63.76, \mathrm{df}=4, \mathrm{p}<0.001\right.$, Nagelkerke $=0.586,81.0 \%$ of all cases were correctly classified with a sensitivity of $77.9 \%$ and a specificity of $87.2 \%$ ). In regression \#1b we examined the 9 key CANTAB tests combined with the CERAD tests and MMSE and found that WLM, VFT and RVP_ML were the most significant predictors $\left(X^{2}=89.73, \mathrm{df}=3\right.$, $\mathrm{p}<0.001$, Nagelkerke $=0.548,82 . \overline{8} \%$ of all cases were correctly classified with a sensitivity of $88.3 \%$ and a specificity of $71.8 \%$ ).

Regression \#2a shows the outcome of a second logistic regression analysis with deficit schizophrenia as the dependent variable and nondeficit schizophrenia as the reference group. We found that SWM-STR and ERT-MORL were significantly and positively associated with deficit schizophrenia whereas PAL-SCFT was negatively related to deficit schizophrenia $\left(\mathrm{X}^{2}=30.69, \mathrm{df}=3, \mathrm{p}<0.001\right.$, Nagelkerke $=0.326,77.6 \%$ of all cases were correctly classified with a sensitivity of $70.3 \%$ and a specificity of $81.0 \%$ ). In regression \#2b we examined the 9 key CANTAB tests combined with the CERAD tests and MMSE and found that True Recall was the only variable discriminating deficit from nondeficit schizophrenia $\left(X^{2}=12.81, \mathrm{df}=1\right.$, $\mathrm{p}<0.001$, Nagelkerke $=0.204,67.5 \%$ of all cases were correctly classified with a sensitivity of $64.9 \%$ and a specificity of $70.0 \%$ ). 
ESF 2, Results, Table 6. Results of 2 different automatic stepwise logistic regression analyses with 1) diagnosis of schizophrenia (SCZ) and 2) deficit schizophrenia (versus nondeficit schizophrenia) as dependent variables.

\begin{tabular}{lllllll}
\hline Dichotomies & $\begin{array}{l}\text { Explanatory } \\
\text { variables }\end{array}$ & Wald & df & p & $\begin{array}{l}\text { Odds } \\
\text { ratio }\end{array}$ & CI 95\% \\
\hline \#1 a SCZ vs HC & RVP_ML & 13.81 & 1 & $<0.001$ & 1.05 & $1.02-1.07$ \\
& SWM_IED & 8.26 & 1 & 0.004 & 1.05 & $1.01-1.08$ \\
& ERT-MORL & 8.15 & 1 & 0.004 & 1.001 & $1.00-$ \\
& Male gender & 6.65 & 1 & 0.010 & 0.20 & $0.06-0.68$ \\
& RVP_ML & 5.89 & 1 & 0.015 & 3.28 & $1.26-8.54$ \\
& VFT & 13.84 & 1 & $<0.001$ & 0.83 & $0.75-0.92$ \\
& WLM & 5.52 & 1 & 0.019 & 0.85 & $0.75-0.96$ \\
\hline \#2a DEFICIT vs & PAL-SCFT & 5.28 & 1 & 0.022 & 0.69 & $0.50-0.95$ \\
NON & & & & & & $1.01-1.23$ \\
& SWM-STR & 4.79 & 1 & 0.029 & 1.12 & $1.00-1.01$ \\
\hline E2b. & ERT-MORL & 4.49 & 1 & 0.034 & 1.00 & $0.47-0.84$ \\
\hline
\end{tabular}

CI 95\%: 95\% confidence interval, lower - upper limit. SCZ vs HC: results of automatic stepwise logistic regression with schizophrenia as dependent variable and no schizophrenia as reference group. DEFICIT vs NON: results of automatic stepwise logistic regression with deficit schizophrenia as dependent variable and no-deficit schizophrenia (controls and nondeficit schizophrenia) as reference group

SWM-BE: Spatial Working Memory, between errors; IED-TT: Intra-Extra Dimensional Set Shift, Total Trials; ERT-MORL: Emotion Recognition Task, mean overall response latency; PAL-SCFT: Paired association learning, stages completed on first trial; SWM-STR: Spatial Working Memory, strategy. 
Acknowledgement

This research has been supported by the Asahi Glass Foundation, Chulalongkorn University Centenary Academic Development Project.

Conflict of interest

The authors have no conflict of interest with any commercial or other association in connection with the submitted article.

Author's contributions

All the contributing authors have participated in the manuscript. MM and BK designed the study. BK recruited patients and completed diagnostic interviews and rating scales measurements. MM carried out the statistical analyses All authors contributed to interpretation of the data and writing of the manuscript.

\section{References}

Al-Dujaili AH, Mousa RF, Al-Hakeim HK, Maes M. High Mobility Group Protein 1 and Dickkopf-Related Protein 1 in Schizophrenia and Treatment-Resistant Schizophrenia: Associations With Interleukin-6, Symptom Domains, and Neurocognitive Impairments. Schizophr Bull. 2020 Sep 24:sbaa136. doi: 10.1093/schbul/sbaa136. Epub ahead of print. PMID: 32971537.

Almulla AF, Al-Hakeim HK, Maes M. Schizophrenia phenomenology revisited: positive and negative symptoms are strongly related reflective manifestations of an underlying single trait indicating overall severity of schizophrenia. CNS Spectr. 2020 May 20:1-10. doi: 10.1017/S1092852920001182. Epub ahead of print. PMID: 32431263.

Andreasen NC. The Scale for the Assessment of Negative Symptoms (SANS): conceptual and theoretical foundations. Brit J Psychiatry Suppl. 1989;7:49-58.

Badcock JC, Michiel PT, Rock D. Spatial working memory and planning ability: contrasts between schizophrenia and bipolar I disorder. Cortex. 2005;41(6):753-63.

Benjamini Y, Hochberg Y. Controlling the false discovery rate: a practical and powerful approach to multiple testing. J Royal Statistics Soc Series b (Methodological). 1995,57,289300 .

Bombin I, Mayoral M, Castro-Fornieles J, Gonzalez-Pinto A, de la Serna E, Rapado-Castro M, Barbeito S, Parellada M, Baeza I, Graell M, Payá B, Arango C. Neuropsychological evidence for abnormal neurodevelopment associated with early-onset psychoses. Psychol Med. 2013;43(4):757-768.

Bonner-Jackson A, Yodkovik N, Csernansky JG, Barch DM. Episodic memory in schizophrenia: the influence of strategy use on behavior and brain activation. Psychiatry Res. 2008;164(1):1-15.

CANTAB. The most validated cognitive research software. http://www.cambridgecognition.com/cantab/ October 1, 2018. 
CERAD. CERAD - An Overview: The Consortium to Establish a Registry for Alzheimer's Disease. 1986; http://cerad.mc.duke.edu/

Eack SM, Wojtalik JA, Newhill CE, Keshavan MS, Phillips ML. Prefrontal cortical dysfunction during visual perspective-taking in schizophrenia. Schizophr Res. 2013;150(23):491-497.

Ferrando PJ, Lorenzo-Seva U. Program FACTOR at 10: Origins, development and future directions. Psicothema. 2017;29:236-240.

Fraser D, Park S, Clark G, Yohanna D, Houk JC. Spatial serial order processing in schizophrenia. Schizophr Res. 2004;70(2-3):203-213.

Hamilton M. A rating scale for depression. J Neurol Neurosurg Psychiatry. 1960;23:56-62.

Harvey PD, Koren D, Reihenberg A, Bowie CR. Negative symptoms and cognitive deficits: What is the nature of their relationship? Schizophr Bull. 2006;32(2):250-258

Hilti CC, Delko T, Orosz AT, Thomann K, Ludewig S, Geyer MA, Vollenweider FX, Feldon J, Cattapan-Ludewig K. Sustained attention and planning deficits but intact attentional setshifting in neuroleptic-naïve first-episode schizophrenia patients. Neuropsychobiology. 2010;61(2):79-86.

Kanchanatawan B, Maes M. The Effects of Tryptophan Catabolites on Negative Symptoms and Deficit Schizophrenia are Partly Mediated by Executive Impairments: Results of Partial Least Squares Path Modeling. CNS Neurol Disord Drug Targets. 2018;17(6):473-486.

Kanchanatawan B, Hemrungrojn S, Thika S, Sirivichayakul S, Ruxrungtham K, Carvalho AF, Geffard M, Anderson G, Maes M. Changes in Tryptophan Catabolite (TRYCAT) Pathway Patterning Are Associated with Mild Impairments in Declarative Memory in Schizophrenia and Deficits in Semantic and Episodic Memory Coupled with Increased FalseMemory Creation in Deficit Schizophrenia. Mol Neurobiol. 2018a;55(6):5184-5201.

Kanchanatawan B, Sriswasdi S, Thika S, Sirivichayakul S, Carvalho AF, Geffard M, Kubera M, Maes M. Deficit schizophrenia is a discrete diagnostic category defined by neuro-immune and neurocognitive features: results of supervised machine learning. Metab Brain Dis. 2018b;33(4):1053-1067.

Kanchanatawan B, Sriswasdi S, Thika S, Stoyanov D, Sirivichayakul S, Carvalho AF, Geffard M, Maes M. Towards a new classification of stable phase schizophrenia into major and simple neuro-cognitive psychosis: Results of unsupervised machine learning analysis. $\mathrm{J}$ Eval Clin Pract. 2018c;24(4):879-891.

Kanchanatawan B, Thika S, Anderson G, Galecki P, Maes M. Affective symptoms in schizophrenia are strongly associated with neurocognitive deficits indicating disorders in executive functions, visual memory, attention and social cognition. Prog Neuropsychopharmacol Biol Psychiatry. 2018d;80(Pt C):168-176. 
Kanchanatawan B, Sriswasdi S, Maes M. Supervised machine learning to decipher the complex associations between neuro-immune biomarkers and quality of life in schizophrenia. Metab Brain Dis. 2019;34(1):267-282.

Kay SR, Fiszbein A, Opler LA. Negative Symptom Rating Scale: limitations in psychometric and research methodology. Psychiatry Res. 1986;19(2):169-173.

Kim HS, An YM, Kwon JS, Shin MS. A preliminary validity study of the Cambridge neuropsychological test automated battery for the assessment of executive function in schizophrenia and bipolar disorder. Psychiatry Investig. 2014;11(4):394-401.

Kirkpatrick B, Buchanan RW, McKenney PD, Alphs LD, Carpenter WT Jr. The Schedule for the Deficit syndrome: an instrument for research in schizophrenia. Psychiatry Res. 1989;30(2):119-123.

Kittirathanapaiboon P, Khamwongpin M. The Validity of the Mini International Neuropsychiatric Interview (M.I.N.I.) Thai Version: Suanprung Hospital, Department of Mental Health, 2005.

Leavitt VM, Goldberg TE. Episodic memory in schizophrenia. Neuropsychol Rev. 2009;19(3):312-23.

Levaux MN, Potvin S, Sepehry AA, Sablier J, Mendrek A, Stip E. Computerized assessment of cognition in schizophrenia: promises and pitfalls of CANTAB. Eur Psychiatry. 2007;22(2):104-115.

Lin IM, Fan SY, Huang TL, Wu WT, Li SM. The Associations between Visual Attention and Facial Expression Identification in Patients with Schizophrenia. Psychiatry Investig. 2013;10(4):393-398.

Lorenzo-Seva U, Ferrando PJ. A General Approach for Fitting Pure Exploratory Bifactor Models. Multivariate Behav Res. 2019;54(1):15-30.

Maes M, Sirivichayakul S, Kanchanatawan B, Vodjani A. Breakdown of the Paracellular Tight and Adherens Junctions in the Gut and Blood Brain Barrier and Damage to the Vascular Barrier in Patients with Deficit Schizophrenia. Neurotox Res. 2019a;36(2):306-322.

Maes M, Vojdani A, Geffard M, Moreira EG, Barbosa DS, Michelin AP, Semeão LO, Sirivichayakul S, Kanchanatawan B. Schizophrenia phenomenology comprises a bifactorial general severity and a single-group factor, which are differently associated with neurotoxic immune and immune-regulatory pathways. Biomol Concepts 2019b;10(1):209-225.

Maes M, Sirivichayakul S, Kanchanatawan B, Carvalho AF. In schizophrenia, psychomotor retardation is associated with executive and memory impairments, negative and psychotic symptoms, neurotoxic immune products and lower natural IgM to malondialdehyde. World $\mathrm{J}$ Biol Psychiatry. 2020a; 7:1-19.

Maes M, Sirivichayakul S, Matsumoto AK, Maes A, Michelin AP, de Oliveira Semeão L, de Lima Pedrão JV, Moreira EG, Barbosa DS, Geffard M, Carvalho AF, Kanchanatawan B. Increased Levels of Plasma Tumor Necrosis Factor- $\alpha$ Mediate Schizophrenia Symptom 
Dimensions and Neurocognitive Impairments and Are Inversely Associated with Natural IgM Directed to Malondialdehyde and Paraoxonase 1 Activity. Mol Neurobiol. 2020b;57(5):23332345.

Maes M, Sirivichayakul S, Matsumoto AK, Michelin AP, de Oliveira Semeão L, de Lima Pedrão JV, Moreira EG, Barbosa DS, Carvalho AF, Solmi M, Kanchanatawan B. Lowered Antioxidant Defenses and Increased Oxidative Toxicity Are Hallmarks of Deficit Schizophrenia: a Nomothetic Network Psychiatry Approach. Mol Neurobiol. 2020c;57(11):4578-4597.

Maes M, Vojdani A, Galecki P, Kanchanatawan B. How to Construct a Bottom-Up Nomothetic Network Model and Disclose Novel Nosological Classes by Integrating Risk Resilience and Adverse Outcome Pathways with the Phenome of Schizophrenia. Brain Sci. 2020d;10(9):645.

O'Leary DS, Flaum M, Kesler ML, Flashman LA, Arndt S, Andreasen NC. Cognitive correlates of the negative, disorganized, and psychotic symptom dimensions of schizophrenia. J Neuropsychiatry Clin Neurosci. 2000;12(1):4-15.

Orellana G, Slachevsky A. Executive functioning in schizophrenia. Front Psychiatry. 2013;4:1-15.

Orellana G, Alvarado L, Muñoz-Neira C, Ávila R, Méndez MF, Slachevsky A. Psychosisrelated matricide associated with a lesion of the ventromedial prefrontal cortex. J Am Acad Psychiatry Law. 2013;41(3):401-406.

Overall JE, Gorham DR. The brief psychiatric rating scale. Psychological Reports. $1962 ; 10: 799-812$

Pantelis C, Barnes TR, Nelson HE, Tanner S, Weatherley L, Owen AM, Robbins TW.Frontal-striatal cognitive deficits in patients with chronic schizophrenia. Brain. 1997;120 ( Pt 10):1823-1843.

Pantelis C, Stuart GW, Nelson HE, Robbins TW, Barnes TR. Spatial working memory deficits in schizophrenia: relationship with tardive dyskinesia and negative symptoms. Am J Psychiatry. 2001;158(8):1276-1285.

Ringle CM, da Silva D, Bido D. Structural equation modeling with the SmartPLS. Brazilian Journal of Marketing - BJM Revista Brasileira de Marketing - ReMark Edição Especial $2014,13, \mathrm{n} 2$.

Roomruangwong C, Matsumoto AK, Michelin AP, de Oliveira Semeão L, de Lima Pedrão JV, Moreira EG, Sirivichayakul S, Carvalho A, Barbosa DS, Maes M. The role of immune and oxidative pathways in menstrual cycle associated depressive, physio-somatic, breast and anxiety symptoms: Modulation by sex hormones. J Psychosom Res. 2020;135:110158.

Rushe TM, Morris RG, Miotto EC, Feigenbaum JD, Woodruff PW, Murray RM. Problemsolving and spatial working memory in patients with schizophrenia and with focal frontal and temporal lobe lesions. Schizophr Res. 1999;37(1):21-33. 
Saleem MM, Harte MK, Marshall KM, Scally A, Brewin A, Neill JC. First episode psychosis patients show impaired cognitive function--a study of a South Asian population in the UK. J Psychopharmacol. 2013;27(4):366-373.

Sánchez P, Peña J, Bengoetxea E, Ojeda N, Elizagárate E, Ezcurra J, Gutiérrez M. Improvements in negative symptoms and functional outcome after a new generation cognitive remediation program: a randomized controlled trial. Schizophr Bull. 2014;40(3):707-715.

Seidman LJ, Lanca M, Kremen WS, Faraone SV, Tsuang MT. Organizational and visual memory deficits in schizophrenia and bipolar psychoses using the Rey-Osterrieth complex figure: effects of duration of illness. J Clin Exp Neuropsychol. 2003;25(7):949-964.

Sewell RA, Perry EB Jr, Karper LP, Bell MD, Lysaker P, Goulet JL, Brenner L, Erdos J, d'Souza DC, Seibyl JP, Krystal JH. Clinical significance of neurological soft signs in schizophrenia: factor analysis of the Neurological Evaluation Scale. Schizophr Res. 2010;124(1-3):1-12.

Sirivichayakul S, Kanchanatawan B, Thika S, Carvalho AF, Maes M. Eotaxin, an Endogenous Cognitive Deteriorating Chemokine (ECDC), Is a Major Contributor to Cognitive Decline in Normal People and to Executive, Memory, and Sustained Attention Deficits, Formal Thought Disorders, and Psychopathology in Schizophrenia Patients. Neurotox Res. 2019a;35(1):122-138.

Sirivichayakul S, Kanchanatawan B, Thika S, Carvalho AF, Maes M. A New Schizophrenia Model: Immune Activation is Associated with the Induction of Different Neurotoxic Products which Together Determine Memory Impairments and Schizophrenia Symptom Dimensions. CNS Neurol Disord Drug Targets. 2019b;18(2):124-140.

Stone M, Gabrieli JD, Stebbins GT, Sullivan EV. Working and strategic memory deficits in schizophrenia. Neuropsychology. 1998;12(2):278-288.

Tamminga, CA, RW Buchanan, JM Gold. The role of negative symptoms and cognitive dysfunction in schizophrenia outcome. Int Clin Psychopharmacol 198;13 (suppl.3),S21-S26

Yu M, Tang X, Wang X, Zhang X, Zhang X, Sha W, Yao S, Shu N, Zhang X, Zhang Z. Neurocognitive Impairments in Deficit and Non-Deficit Schizophrenia and Their Relationships with Symptom Dimensions and Other Clinical Variables. PLoS One. 2015;10(9):e0138357.

WHO. Study protocol for the World Health Organization project to develop a Quality of Life assessment instrument (WHOQOL). Qual Life Res. 1993,2(2),153-159.

Zilles D, Gruber E, Falkai P, Gruber O. Patients with schizophrenia show deficits of working memory maintenance components in circuit-specific tasks. Eur Arch Psychiatry Clin Neurosci. 2010;260(7):519-525. 